Universidade de São Paulo

Instituto de Física de São Carlos

Prof. Dr. Daniel Augusto Turolla Vanzella

\title{
Efeitos de vácuo em espaços-tempos curvos
}

Tese apresentada ao Instituto de Física de São Carlos da Universidade de São Paulo para obtenção do título de Livre Docente.

São Carlos

2015 

A meus filhos, Lucca e Matteo 



\section{AGRADECIMENTOS}

Aos meus colaboradores e amigos George E. A. Matsas, William C. C. de Lima,

André G. S. Landufo e Raissa F. P. Mendes, pelas discussões inestimáveis sobre teoria de campos em espaços curvos e, em particular, sobre o efeito do despertar do vácuo, que constitui o tema central desta tese.

Ao Prof. Luiz Nunes de Oliveira, por me incentivar a escrever esta tese para perseguir o título de Livre Docente;

À secretária do Grupo de Física Teórica do FCI-IFSC, Cristina Ligo Silva, pela eficiência e solicitude sempre que preciso de sua ajuda, e pela compreensão e paciência diante de minhas requisições de última hora;

Aos funcionários da gráfica do IFSC, Aurotides, Denise e Italo, pela presteza e rapidez em cumprir meus prazos apertados;

Ao meu amigo Guilherme Innocentini, pelo incentivo e ajuda com a formatação desta tese;

À minha amada família, Aline, Lucca e Matteo, por dar sentido à minha vida e motivação para perseguir meus objetivos. 



\section{RESUMO}

VANZELLA D. A. T. Efeitos de vácuo em espaços-tempos curvos. 2015. 52 p. Tese de Livre Docência - Instituto de Física de São Carlos, Universidade de São Paulo, São Carlos, 2015.

As idéias e análises apresentados nesta tese são parte do fruto de anos de investigação envolvendo aspectos fundamentais de teoria quântica de campos em espaçostempos curvos. O foco será o efeito da dominância do vácuo induzida pela gravitação, também chamado de despertar do vácuo, segundo o qual algumas evoluções bem comportadas da geometria do espaço-tempo de fundo são capazes de desencadear um crescimento exponencial da densidade de energia de vácuo de certos campos, de modo a torná-la protagonista na evolução subsequente do sistema. Apresentaremos o status atual de nosso entendimento acerca desse efeito e discutiremos perspectivas de investigações futuras (algumas já em andamento).

Palavras-chave: Gravitação. Energia de vácuo. 



\begin{abstract}
VANZELLA D. A. T. Vacuum effects in curved spacetimes. 2015. 52 p. Tese de Livre Docência - Instituto de Física de São Carlos, Universidade de São Paulo, São Carlos, 2015.

The ideas and analyses presented in this thesis represent part of the results of many years of investigations concerning fundamental aspects of quantum field theory in curved spaceitimes. We shall focus on the gravity-induced vacuum dominance, or vacuum awakening effect, according to which well-behaved background geometries can trigger an exponential growth of the vacuum energy density of some fields, forcing the latter to become relevant or even dominant to the subsequent evolution of the system. We shall discuss the present status of our understanding concerning this effect and point to future perspectives (some of them presently under investigation).
\end{abstract}

Keywords: Gravitation. Vacuum energy. 



\section{SUMÁRIO}

1 Introdução 11

1.1 Energia de vácuo em espaço plano: escondida em plena vista . . . . . 13

1.2 Energia de vácuo em espaços curvos . . . . . . . . . . . . . 16

2 Teoria Quântica de Campos em Espaços Curvos 19

2.1 Formalismo canônico de TQCEC . . . . . . . . . . . . . . 21

2.2 Valor esperado do tensor energia-momento e estados Hadamard . . . 27

3 Despertar do vácuo por campos gravitacionais 31

3.1 Dominância do vácuo induzida pela gravitação . . . . . . . . . . . . 32

3.2 Despertando o vácuo em estrelas relativísticas . . . . . . . . . . 38

3.3 Restabilização e produção de partículas . . . . . . . . . . . 43

3.4 De flutuações quânticas a perturbações clássicas . . . . . . . . . . . 47

4 Comentários finais 49

REFERÊNCIAS 


\section{Capítulo 1}

\section{Introdução}

Numa apresentação à Sociedade Alemã de Física, em 14 de dezembro de 1900, M. Planck lançava as bases, sem saber, do que viria a ser uma revolução em nosso entendimento da Natureza em sua escala mais íntima. O problema de interesse de Planck, então, era o da radiação de corpo negro. Essa radiação, com propriedades universais, pode ser modelada pela radiação em equilíbrio no interior de uma cavidade de paredes metálicas mantidas a uma temperatura $T$ constante. Embora os detalhes dos modos normais da radiação (ou seja, do campo eletromagnético) no interior da cavidade dependam da geometria desta última, podemos considerar, por simplicidade, uma cavidade cúbica de dimensões $L \times L \times L$ para fazer uma contagem de modos, obtendo que, para frequências (angulares) $\omega$ tais que $\omega / c \gg 1 / L$ ( $c$ é a velocidade da luz no vácuo), o número de modos normais da radiação com frequência (angular) entre $\omega$ e $\omega+d \omega$ é dado por (veja, por exemplo, Ref. [1):

$$
d N=n(\omega) d \omega=\frac{V \omega^{2}}{\pi^{2} c^{3}} d \omega
$$

onde $n(\omega)$ é a densidade espectral dos modos normais na cavidade e $V=L^{3}$. $^{1}$ Como os modos normais podem ser encarados como os graus de liberdade da radiação, independentes entre si, a hipótese de equilíbrio térmico dessa radiação com as paredes

\footnotetext{
${ }^{1} \mathrm{O}$ regime $\omega / c \gg 1 / L$ é útil para podermos aproximar a densidade espectral $n(\omega)$ por uma função contínua. A rigor, $n(\omega)$ é uma distribuição cujo suporte é um subconjunto discreto da reta real (o espectro de frequências de um problema de contorno numa região finita).
} 
da cavidade e a aplicação do "teorema da equipartição de energia" a esse sistema levaria à conclusão de que a densidade de energia no interior da cavidade, armazenada em modos com frequência entre $\omega$ e $\omega+d \omega$, seria dada por

$$
d \rho=k_{B} T \frac{d N}{V}=\frac{k_{B} T \omega^{2}}{\pi^{2} c^{3}} d \omega
$$

onde $k_{B}$ é a constante de Boltzmann. Isso, por sua vez, levaria à consequência absurda de que a densidade de energia total dentro da cavidade (ou seja, considerandose todos os modos possíveis) é infinita, pois a densidade de energia armazenada em modos com frequência até $\omega$ cresceria proporcionalmente a $\omega^{3}$. Essa discrepância grave entre a realidade e a predição teórica desse modelo ficou conhecida como $c a$ tástrofe do ultra-violeta, uma referência direta ao regime de frequências responsável por tal discrepância.

Para lidar com esse problema, Planck postulou que a troca de energia entre a radiação e as paredes da cavidade só poderia se dar de maneira discreta, pela troca de quanta de energia cujo valor $\epsilon(\omega)$ dependia da frequência $\omega$ da radiação segundo

$$
\epsilon(\omega)=\hbar \omega
$$

onde $\hbar$ é uma constante. Assim sendo, ao invés de um dado modo normal com frequência $\omega$ poder carregar qualquer valor de energia $\mathcal{E} \in \mathbb{R}_{+}$(o que, combinado com a distribuição de Boltzmann, leva ao valor médio $\langle\mathcal{E}\rangle=k_{B} T$ do teorema de equipartição utilizado na argumentação anterior), apenas valores $n \epsilon(\omega)$, com $n \in$ $\mathbb{N}$, são permitidos. A energia média por modo normal em equilíbrio térmico à temperatura $T \equiv 1 /\left(k_{B} \beta\right)$ passa a ser, então,

$$
\langle\mathcal{E}\rangle=\frac{1}{Z} \sum_{n=0}^{\infty} n \epsilon(\omega) e^{-\beta n \epsilon(\omega)}=\frac{\epsilon(\omega)}{e^{\beta \epsilon(\omega)}-1},
$$

onde

$$
Z:=\sum_{n=0}^{\infty} e^{-\beta n \epsilon(\omega)}=\frac{1}{1-e^{-\beta \epsilon(\omega)}}
$$

nada mais é que o fator de normalização para a distribuição de Boltzmann mas agora 
aplicada a valores discretos de energia possíveis. De (1.1) e (1.4) tem-se, então, para a densidade de energia total no interior da cavidade

$$
\rho=\int \frac{d N}{V}\langle\mathcal{E}\rangle=\frac{\hbar}{\pi^{2} c^{3}} \int_{0}^{\infty} d \omega \frac{\omega^{3}}{e^{\beta \hbar \omega}-1}=\frac{\pi^{2} k_{B}^{4} T^{4}}{15 \hbar^{3} c^{3}} .
$$

Esse resultado não só é finito como leva à lei de Stefan-Boltzmann verificada experimentalmente para a intensidade da radiação de corpo negro como função da temperatura: $I=\sigma T^{4}=\rho c / 4$, onde $\sigma$ é a constante de Stefan-Boltzmann medida experimentalmente, de onde se pode extrair um valor para a constante de Planck $\hbar$.

Em resumo, a quantização da energia da radiação segundo a Eq. (1.3) (seja devido à troca discreta com as paredes, seja pela quantização da própria radiação, como proposta por A. Einstein cinco anos depois) faz com que os modos normais

com frequência $\omega \gg k_{B} T / \hbar$ sejam exponencialmente suprimidos pelo fator de Boltzmann $e^{-\beta \hbar \omega}$, ficando "congelados", impedidos de serem excitados a não ser muito raramente. Esse é o mecanismo pelo qual o comportamento ultra-violeta da radiação é domado, evitando a catástrofe do tratamento clássico.

Com isso em mente, é irônico pensar que o próprio procedimento de quantização, levado adiante e implementado de maneira completa para campos algumas décadas mais tarde, faria com que o regime ultra-violeta voltasse a assombrar o cálculo de observáveis físicos.

\subsection{Energia de vácuo em espaço plano: escondida em plena vista}

Uma maneira formal (i.e., sem preocupação demasiada com rigor) de se levar adiante o procedimento de quantização de um sistema clássico, descrito por coordenadas canônicas $q_{j}$ e respectivos momentos canonicamente conjugados $p_{j}$ ( $j$ pertencendo a algum conjunto de índices $\mathcal{I}$ ), é "promovê-los" a (i.e., mapeá-los em) operadores auto-adjuntos $\hat{q}_{j}$ e $\hat{p}_{j}$, respectivamente, satisfazendo as relações de comutação de 
Heisenberg $(\mathrm{RCH})$

$$
\left[\hat{q}_{j}, \hat{p}_{k}\right]=i \hbar \delta_{j k}
$$

onde $i$ é a unidade imaginária e $\delta_{j k}$ é a distribuição $\delta$-de-Dirac apropriada para o conjunto $\mathcal{I}$. Estados do sistema físico são, então, caracterizados por estados algébricos, ou seja, funcionais lineares positivos e normalizados sobre a álgebra gerada por $\hat{q}_{j}$ e $\hat{p}_{j}$ (vide, por exemplo, Ref. [2]). A conexão com o mundo físico é feita pela imposição de que se o sistema se encontrar no estado $\ell$, então o valor esperado (ou seja, a média) de medições feitas do observável $A$ (mapeado no operador auto-adjunto $\hat{A})$ sobre o sistema nesse estado, $\langle A\rangle_{\ell}$, é dado por

$$
\langle A\rangle_{\ell} \equiv \ell(\hat{A})
$$

Da positividade e linearidade de qualquer estado e da relação algébrica (1.7) segue que

$$
\left\langle q_{j}^{2}\right\rangle\left\langle p_{k}^{2}\right\rangle \geq \frac{\hbar^{2}}{4} \delta_{j k}
$$

(O subescrito em \langle\rangle será suprimido sempre que estiver claro, pelo contexto, qual é o estado em que o valor esperado está sendo calculado ou quando for um resultado válido para qualquer estado, como o acima.) Dessa maneira, se a energia carregada pelo grau de liberdade descrito por $\left(q_{j}, p_{j}\right)$ for da forma $\mathcal{E}_{j}=p_{j}^{2} / 2+c_{j}^{2} q_{j}^{2} / 2$, como acontece com muitos sistemas simples de interesse ( $c_{j}$ são constantes reais), então (1.9) impõe que haja uma energia mínima que esse grau de liberdade pode armazenar no regime quântico:

$$
\left\langle\mathcal{E}_{j}\right\rangle \geq \frac{\hbar c_{j}}{2}
$$

A mensagem aqui é importante: um grau de liberdade desse sistema nunca pode estar totalmente "congelado" nem, tampouco, arbitrariamente suprimido. Devemos, portanto, revisitar a análise da radiação de corpo negro feita anteriormente, onde os modos com frequência arbitrariamente alta eram arbitrariamente suprimidos. 
O estado particular $\ell_{0}$ que satura a desigualdade 1.10 para todo $j \in \mathcal{I}$ é definido como sendo o estado de vácuo do sistema. Nesse estado, cada grau de liberdade possui uma flutuação de ponto zero de suas variáveis canônicas dada por $\left\langle q_{j}^{2}\right\rangle_{\ell_{0}}=\hbar /\left(2\left|c_{j}\right|\right)$ e $\left\langle p_{j}^{2}\right\rangle_{\ell_{0}}=\hbar\left|c_{j}\right| / 2$, que, por sua vez, armazena uma energia de ponto zero $\left\langle\mathcal{E}_{j}\right\rangle_{\ell_{0}}=\hbar c_{j} / 2$. Adaptando esses resultados para os modos normais da radiação numa cavidade, onde $q_{j}$ são associados à amplitude de oscilação dos modos normais e $p_{j}$ à amplitude de oscilação da derivada temporal, tem-se que $c_{j}=\omega_{j}$ (a frequência dos modos normais) e a densidade de energia de vácuo é dada por

$$
\langle\rho\rangle_{\ell_{0}}=\frac{1}{V} \int_{\mathcal{I}} d \mu(j) n(j)\left\langle\mathcal{E}_{j}\right\rangle_{\ell_{0}}=\frac{\hbar}{2 \pi^{2} c^{3}} \int_{0}^{\infty} d \omega \omega^{3},
$$

onde $n(j)$ é a densidade espectral de modos e $\mu$ é uma medida sobre $\mathcal{I}$. Evidentemente, o resultado é divergente devido ao limite superior de integração; a catástrofe do ultra-violeta parece ter sido vingada! No caso da radiação em equilíbrio térmico à temperatura $T$ no interior da cavidade, basta trocar $n$ por $n+1 / 2$ nas manipulações em (1.4) e 1.5 para se obter a nova densidade de energia desse estado térmico $\ell_{T}$ :

$$
\langle\rho\rangle_{\ell_{T}}=\frac{\hbar}{\pi^{2} c^{3}} \int_{0}^{\infty} d \omega \omega^{3}\left(\frac{1}{e^{\beta \hbar \omega}-1}+\frac{1}{2}\right) .
$$

Nota-se prontamente que o novo resultado nada mais é do que o resultado anterior, Eq. (1.6), somado à contribuição de vácuo, Eq. (1.11)

Devemos recordar, aqui, que os dados observacionais da radiação de corpo negro são perfeitamente bem explicados pela primeira parcela da Eq. 1.12). Se acreditamos, portanto, neste último resultado, as medições experimentais devem ser cegas à contribuição (divergente) do vácuo. A explicação normalmente utilizada para isso é que valores absolutos de (densidade de) energia não tem significado físico, podendo ser redifinidos por uma constante aditiva arbitrária, o que, por outro lado, não altera variações de energia, estas sim providas de significado. Em outras palavras, o vácuo pode ser tomado como o estado fiducial em relação ao qual as energias de todos os outros estados são medidas. 
Isso não significa, no entanto, que a energia de vácuo não tenha efeitos observáveis. Como dito anteriormente, os detalhes dos modos normais de um campo dentro de uma cavidade dependem da geometria desta última. Sendo assim, uma mudança na geometria da cavidade pode afetar os valores das possíveis frequências $\omega_{j}$, fazendo com que a energia de vácuo em seu interior mude. Isso acarreta um efeito mensurável: o aparecimento de uma força sobre as paredes da cavidade tentando favorecer uma geometria que minimize essa energia. A existência dessa força, chamada de efeito Casimir [3], é, de fato, verificada experimentalmente em laboratório [4]. O efeito Casimir denuncia a realidade física das flutuações de ponto zero, a despeito da divergência da expressão 1.11 não ter consequências mais graves. Pelo menos enquanto gravitação não é considerada.

\subsection{Energia de vácuo em espaços curvos}

Há cem anos, A. Einstein apresentava ao mundo o fruto de seus quase dez anos de esforços tentando incorporar a gravitação newtoniana ao paradigma de sua Teoria da Relatividade de 1905. O resultado desses esforços foi uma teoria da gravidade que revolucionou, mais uma vez, nosso entendimento de espaço e tempo: não só tempo e espaço eram facetas relativas (i.e., dependentes de observador) de um ente mais fundamental e absoluto, o espaço-tempo, como a geometria desse espaço-tempo era uma propriedade dinâmica, passível de ser "distorcida" pela presença de energia (e momento, pressão e estresse). Nesse novo paradigma, que ficou conhecido como Relatividade Geral (RG), a gravitação é considerada como a manifestação dessa deformação e suas consequências sobre os corpos e campos que evoluem nesse espaçotempo curvo de fundo (veja, por exemplo, Ref. [5]).

A equação dinâmica proposta por Einstein, que leva seu nome, é escrita de forma compacta como

$$
G_{\mu \nu}:=R_{\mu \nu}-\frac{1}{2} g_{\mu \nu} R=\frac{8 \pi G}{c^{4}} T_{\mu \nu},
$$


onde o tensor de Einstein $G_{\mu \nu}$ é definido pela expressão que segue a ele acima, $R_{\mu \nu}$ é o tensor de curvatura de Ricci, que depende da métrica $g_{\mu \nu}$ do espaço-tempo, $R:=g^{\mu \nu} R_{\mu \nu}$ (onde $g^{\mu \nu}$ é o inverso da métrica $g_{\mu \nu}$ ) e, do lado direito da equação, $G$ é a constante da gravitação de Newton e $T_{\mu \nu}$ é o tensor energia-momento total do sistema físico (de partículas ou campos) causando a distorção do espaço-tempo. Colocando de uma maneira simplista, a equação de Einstein dita como a presença de energia (e momento, pressão e estresse), codificada no lado direito da Eq. (1.13), influencia a geometria do espaço-tempo de fundo, codificada predominantemente nos tensores geométricos do lado esquerdo de (1.13) mas também, implícita ou explicitamente, em $T_{\mu \nu}{ }^{2}$. Mas essa equação faz mais: ela também impõe vínculos sobre a evolução do sistema descrito por $T_{\mu \nu}$ (e, em alguns casos simples, até mesmo determina essa evolução). Isso se dá como consequência da identidade de Bianchi [5], que implica na identidade geométrica $\nabla_{\mu} G^{\mu \nu} \equiv 0$ que, por sua vez, leva a

$$
\nabla_{\mu} T^{\mu \nu}=0
$$

se a equação de Einstein é satisfeita. Essa é a expressão covariante da conservação local de energia e momento válida para espaços-tempos curvo\$3 ${ }^{3}$ Resumindo, nas palavras de J. Wheeler [6], a matéria diz ao espaço-tempo como se curvar [através de (1.13)] e o espaço-tempo diz à matéria como se mover [satisfazendo (1.14]).

Uma característica importante da gravitação, assim como regida pela equação de Einstein, é que ela é sensível ao valor da (densidade de) energia do sistema, e não apenas a diferenças de energia. Desse modo, podemos antecipar que o procedimento de quantização de campos levada a cabo em espaços-tempos curvos, que é o foco da Teoria Quântica de Campos em Espaços-tempos Curvos (TQCEC), leva a reflexões

\footnotetext{
22mbora a Eq. 1.13 seja uma equação diferencial parcial (EDP) para o campo $g_{\mu \nu}$, há uma diferença importante entre essa equação e outras equações de campo: a presença de $g_{\mu \nu}$ no termo de "fonte". Isso faz com que, diferentemente do eletromagnetismo, por exemplo, onde podemos resolver para o campo eletromagnético para uma corrente dada, a forma exata da fonte de gravitação $T_{\mu \nu}$ só seja determinada a posteriori.

${ }^{3}$ Em geral, conservações globais não se verificam, a menos que haja simetrias da geometria do espaço-tempo de fundo.
} 
sobre a energia de vácuo que até agora podíamos evitar.

A organização desta tese é a seguinte. No Capítulo 2 apresentaremos, de maneira breve, o formalismo de TQCEC, focando-nos nos aspectos conceituais e operacionais que serão utilizados mais adiante. Em particular, justificaremos o porquê estamos interessados em efeitos de vácuo que não advêm do regime ultra-violeta dos campos. No Capítulo 3 apresentaremos o tema principal desta tese: o efeito do despertar do vácuo. Por fim, no Capítulo 44 faremos alguns comentários finais e apontaremos questões interessantes ainda a serem analisadas e que constituem linhas de investigação que devem ser seguidas futuramente (algumas já em andamento). 


\section{Capítulo 2}

\section{Teoria Quântica de Campos em}

\section{Espaços Curvos}

Nascida dos esforços de se conciliar os dois pilares sobre os quais a Física Moderna assentava-se até meados do século passado, a Teoria Quântica de Campos (TQC) conseguiu incorporar, num único formalismo, as duas mais profundas revoluções ocorridas no âmbito das teorias físicas desde o advento dos Principia de I. Newton: a Teoria da Relatividade (Restrita) e a Mecânica Quântica. Deslocando o enfoque de partículas para campos como os objetos fundamentais da Natureza, a TQC provê o instrumental adequado para a descrição de três das quatro interações fundamentais conhecidas, a saber, das interações eletromagnética e nuclear fraca, unificadas na chamada teoria eletrofraca, e da interação nuclear forte, descrição esta conhecida como cromodinâmica quântica.

De outro lado, a teoria da Relatividade Geral (RG), que descreve a interação gravitacional como uma teoria clássica de campo, resiste como sendo a última teoria clássica ainda utilizada, com sucesso inigualado por uma versão quântica, para descrever uma interação fundamental. Não se conhece, até o momento, nenhum dado observacional confiável para o qual RG forneça uma predição dissonante. Porém, a despeito de seu maiúsculo sucesso, considerações teóricas levam-nos a crer que a 
RG é apenas uma teoria efetiva, válida, como uma ótima aproximação, em escalas "macroscópicas". Por "macroscópicas", aqui, entende-se escalas de espaço e tempo muito maiores que $l_{P}:=\left(G \hbar / c^{3}\right)^{1 / 2} \approx 10^{-33} \mathrm{~cm}$ (comprimento de Planck) e $t_{P}:=$ $l_{P} / c \approx 10^{-43} \mathrm{~s}$ (tempo de Planck), respectivamente. Na escala de Planck, acreditase que a descrição fornecida pela RG seja inadequada, havendo a necessidade de uma teoria quântica da gravidade. Contudo, para a frustração de gerações de físicos teóricos, a mais quotidiana das interações fundamentais tem resistido a todas as tentativas de descrição coerente em termos de uma TQC.

Como um passo intermediário na busca de uma teoria quântica da gravidade, a Teoria Quântica de Campos em Espaços Curvos (TQCEC), também conhecida como gravitação semi-clássica, pode ser encarada como a "fusão" mínima entre os formalismos de TQC e RG, possuindo, por isso mesmo, um embasamento sólido e confiável. Em TQCEC, os campos de matéria (ou das outras interações) são quantizados sobre a geometria clássica do espaço-tempo (curvo) subjacente, implementando, dessa forma, a influência exercida pela interação gravitacional sobre os fenômenos quânticos descritos por TQC. Sua origem remonta o final da década de 60 do último século, quando L. Parker e Ya.B. Zel'dovich, independentemente, desenvolveram um formalismo, com motivações cosmológicas, com a finalidade de analisar a criação de partículas em campos gravitacionais intensos. (Para uma revisão abrangente de TQCEC, vide Refs. [7]

O mais conhecido efeito de TQCEC já descoberto é o chamado efeito Hawking, também conhecido como "evaporação de buracos negros". Utilizando a teoria clássica de RG [10], juntamente com hipóteses bastante "razoáveis" acerca das propriedades da matéria, S. Hawking e R. Penrose haviam provado um conjunto de teoremas cuja implicação era que buracos negros seriam objetos indestrutíveis: a área do horizonte de eventos de cada buraco negro individualmente não poderia, por nenhum processo, ser reduzida, o mesmo valendo para a soma das áreas dos horizontes de eventos de todos os buracos negros no Universo. No entanto, o próprio Hawking [11], pouco 
tempo depois, mostrou que cálculos de TQCEC aplicada a buracos negros, ou seja, a quantização de campos de matéria no espaço-tempo de estrelas colapsando, previa a existência de um fluxo térmico de partículas sendo emitido pelo buraco, com uma temperatura característica dada por $T_{H}=\hbar c^{3} /\left(8 \pi k_{B} G M\right) \approx 6 \times 10^{-8}\left(M_{\odot} / M\right) \mathrm{K}$, com $M$ sendo a massa do buraco negro e $M_{\odot} \approx 2 \times 10^{30} \mathrm{~kg}$ a massa do Sol. Em consequência desse fluxo térmico, a massa do buraco negro, e consequentemente sua área, deve diminuir no processo. Se essa redução continua até que o buraco negro desapareça por completo ou é interrompida quando o seu tamanho atinge a escala de Planck é uma questão em aberto até o momento. Mas de qualquer maneira, o efeito Hawking mostrou que buracos negros não são tão "negros" e indestrutíveis afinal.

A seguir faremos uma apresentaremos sucinta do formalismo canônico de TQCEC. Ao longo de todo o texto, a partir deste ponto, faremos uso de unidades em que $\hbar=G=c=k_{B}=1$, a menos que explicitamente dito o contrário. Também faremos uso da notação abstrata de índices introduzida na Ref. [5] para representar objetos e equações tensoriais.

\subsection{Formalismo canônico de TQCEC}

Seja $\mathcal{M}$ uma variedade diferenciável (Hausdorff e paracompacta [5, 10]) e $g_{a b}$ uma métrica lorentziana definida em $\mathcal{M}$ de modo que o espaço-tempo $\left(\mathcal{M}, g_{a b}\right)$ seja globalmente hiperbóliç $\bigsqcup_{1}^{1}$ Nesse espaço-tempo, consideremos um campo (escalar, por simplicidade) livr $£^{2} \Phi$ cuja ação $S_{m}$ seja dada por

$$
S_{m}\left[g_{a b}, \Phi\right]=-\frac{1}{2} \int_{\mathcal{M}} d^{4} x \sqrt{-g}\left[\nabla^{a} \Phi \nabla_{a} \Phi+\left(m^{2}+\xi R\right) \Phi^{2}\right]
$$

onde $g:=\operatorname{det}\left(g_{\mu \nu}\right)$ no sistema de coordenadas usado para expressar o elemento de integração $d^{4} x, m$ é a massa do campo e $\xi \in \mathbb{R}$ é uma constante de acoplamento

\footnotetext{
${ }^{1}$ Essa condição é para garantir que equações de campo hiperbólicas sobre $\left(\mathcal{M}, g_{a b}\right)$ determinem problemas de Cauchy bem-postos.

2"Livre" nesse contexto significa sem interações além da gravitacional.
} 
do campo com o escalar de curvatura $R$ do espaço-tempo. A equação clássica de campo advinda da estacionaridade de $S_{m}$ por variações de $\Phi, \delta S_{m} /\left.\delta \Phi\right|_{\Phi=\phi}=0$, é dada por

$$
-\square \phi+\left(m^{2}+\xi R\right) \phi=0,
$$

onde $\square:=g^{a b} \nabla_{a} \nabla_{b}$. Essa é a chamada equação de Klein-Gordon. Além disso, de $S_{m}$ também se tira o tensor energia-momento do campo $\Phi$ :

$$
\begin{aligned}
T_{a b}:=-\frac{2}{\sqrt{-g}} \frac{\delta S_{m}}{\delta g^{a b}}= & (1-2 \xi) \nabla_{a} \Phi \nabla_{b} \Phi+\xi R_{a b} \Phi^{2}-2 \xi \Phi \nabla_{a} \nabla_{b} \Phi \\
& +(2 \xi-1 / 2) g_{a b}\left[\nabla^{c} \Phi \nabla_{c} \Phi+\left(m^{2}+\xi R\right) \Phi^{2}\right] .
\end{aligned}
$$

A hiperbolicidade global de $\left(\mathcal{M}, g_{a b}\right)$ implica que o espaço-tempo pode ser foliado, $\mathcal{M}=\mathbb{R} \times \Sigma$, onde cada $\Sigma_{t}:=\{t\} \times \Sigma$, com $t \in \mathbb{R}$, é uma sub-variedade tridimensional tipo-espaço segundo $g_{a b}$. Além disso, cada $\Sigma_{t}$ é uma superfície de Cauchy do espaçotempo, de modo que condições iniciais "apropriadas" fornecidas sobre uma dada $\Sigma_{t}$ qualquer, determinam soluções da Eq. (2.2) em todo o espaço-tempo. Fazendo uso dessa foliação de $\mathcal{M}$, podemos adotar $t$ como uma das coordenadas e sobre cada $\Sigma_{t}$ escolher coordenadas $x^{i}, i=1,2,3$, de modo que $n^{a} \nabla_{a} x^{i}=0$, onde $n^{a}$ é um campo vetorial unitário $\left(n^{a} n_{a}=-1\right)$ ortogonal a todo $\Sigma_{t}$. Nessas coordenadas, o elemento de linha toma a forma

$$
d s^{2}=g_{\mu \nu} d x^{\mu} d x^{\nu}=N^{2}\left(-d t^{2}+h_{i j} d x^{i} d x^{j}\right)
$$

onde $N \equiv N(x)>0$ e $h_{i j} \equiv h_{i j}(x)$, com $x=\left(t, x^{i}\right)$. Com isso, podemos rescrever a ação dada acima como

$$
S_{m}\left[g_{a b}, \phi\right]=\frac{1}{2} \int_{\mathbb{R}} d t \int_{\Sigma_{t}} d^{3} x \sqrt{{ }^{(3)} g}\left[N^{-1}\left(\dot{\Phi}^{2}-h^{i j} \nabla_{i} \phi \nabla_{j} \phi\right)-N\left(m^{2}+\xi R\right) \phi^{2}\right]
$$

onde $\dot{\Phi}:=\partial \Phi / \partial t, h^{i j}$ é a inversa de $h_{i j}$ e ${ }^{(3)} g=\operatorname{det}\left({ }^{(3)} g_{i j}\right)$, onde ${ }^{(3)} g_{i j}=N^{2} h_{i j}$ é a métrica riemanniana induzida em cada $\Sigma_{t}$. 
De acordo com o formalismo canônico, o momento conjugado a $\Phi$ é dado por

$$
\Pi:=\frac{\delta S_{m}}{\delta \dot{\Phi}}=\sqrt{{ }^{(3)} g} N^{-1} \dot{\Phi}=\sqrt{{ }^{(3)} g} n^{a} \nabla_{a} \Phi
$$

Essas variáveis canônicas devem ser representadas por (distribuições de) operadores auto-adjuntos $\hat{\Phi}$ e $\hat{\Pi}$ satisfazendo as relações de comutação de Heisenberg apropriadas para campos:

$$
\left[\hat{\Phi}\left(t, x^{i}\right), \hat{\Pi}\left(t, x^{i}\right)\right]=i \delta^{(3)}\left(x^{i}-x^{i}\right)
$$

Para contruir uma representação satisfazendo essas relações, considere uma base do espaço $\mathcal{S}_{\mathbb{C}}$ de soluções complexificado da Eq. (2.2), $\left\{u_{\alpha}, u_{\alpha}^{*}\right\}_{\alpha \in \mathcal{J}}$, satisfazendo

$$
\begin{gathered}
\left(u_{\alpha}, u_{\beta}\right)_{K G}=-\left(u_{\alpha}^{*}, u_{\beta}^{*}\right)_{K G}=\delta(\alpha, \beta), \\
\left(u_{\alpha}, u_{\beta}^{*}\right)_{K G}=0
\end{gathered}
$$

onde $(,)_{K G}$ é o produto interno de Klein-Gordon, definido por

$$
(f, g)_{K G}:=i \int_{\Sigma_{t}} d^{3} x \sqrt{{ }^{(3)} g} n^{a}\left(f^{*} \nabla_{a} g-g \nabla_{a} f^{*}\right)
$$

e $\delta$ é a distribuição $\delta$-de-Dirac definida em $\mathcal{J}$. Seja $\mathcal{S}_{\mathbb{C}}^{+} \subset \mathcal{S}_{\mathbb{C}}$ o subespaço gerado por $\left\{u_{\alpha}\right\}_{\alpha \in \mathcal{J}}$. Munido do produto interno de Klein-Gordon, $\mathcal{S}_{\mathbb{C}}^{+}$pode ser completado para se obter um espaço de Hilbert $\mathcal{H}$. Com esse espaço de Hilbert em mãos, construimos o espaço de Fock (simétrico) associado:

$$
\mathcal{F}_{s}(\mathcal{H}):=\oplus_{n=0}^{\infty}\left(\mathcal{H}^{\otimes n}\right)_{s} \equiv \mathbb{C} \oplus \mathcal{H} \oplus(\mathcal{H} \otimes \mathcal{H})_{s} \oplus(\mathcal{H} \otimes \mathcal{H} \otimes \mathcal{H})_{s} \oplus \ldots
$$

Um elemento arbitrário desse espaço tem a forma

$$
|\Psi\rangle=\left(\psi_{0}, \psi_{1}, \psi_{2}, \psi_{3}, \ldots\right)
$$

onde cada $\psi_{n}$ é uma função de $n$ pontos do espaço-tempo, simétrica em seus argumentos, gerada pelo produto simetrizado das funções $u_{\alpha}$. Além disso, $|\Psi\rangle$ deve 
satisfazer

$$
(|\Psi\rangle,|\Psi\rangle) \equiv\langle\Psi \mid \Psi\rangle:=\left|\psi_{0}\right|^{2}+\left\|\psi_{1}\right\|^{2}+\left\|\psi_{2}\right\|^{2}+\left\|\psi_{3}\right\|^{2}+\ldots<\infty
$$

onde o produto interno em cada $\mathcal{H}^{\otimes n}, n \in \mathbb{N}^{*}$, é definido pela maneira como atua na base,

$$
\left(u_{\alpha_{1}} \otimes \ldots \otimes u_{\alpha_{n}}, u_{\beta_{1}} \otimes \ldots \otimes u_{\beta_{n}}\right):=\left(u_{\alpha_{1}}, u_{\beta_{1}}\right)_{K G} \ldots\left(u_{\alpha_{n}}, u_{\beta_{n}}\right)_{K G}
$$

e estendido por linearidade e fecho para todo $\mathcal{H}^{\otimes n}$.

Agora definiremos em $F_{s}(\mathcal{H})$ duas famílias de operadores: $\hat{a}_{\alpha}$ e $\hat{a}_{\alpha}^{\dagger}, \alpha \in \mathcal{J}$. Novamente, isso será feito pela maneira como atuam na base de cada $\left(\mathcal{H}^{\otimes n}\right)_{s}$ :

$$
\hat{a}_{\alpha} u_{\left(\alpha_{1}\right.} \otimes \ldots \otimes u_{\left.\alpha_{n}\right)}:=\sqrt{n}\left(u_{\alpha}, u_{\left(\alpha_{1}\right.}\right)_{K G} u_{\alpha_{2}} \otimes \ldots \otimes u_{\left.\alpha_{n}\right)}, \quad n \in \mathbb{N}^{*},
$$

enquanto que $\hat{a}_{\alpha}$ atuando em $\mathbb{C} \subset F_{s}(\mathcal{H})$ é o operador nulo; e

$$
\hat{a}_{\alpha}^{\dagger} u_{\left(\alpha_{1}\right.} \otimes \ldots \otimes u_{\left.\alpha_{n}\right)}:=\sqrt{n+1} u_{(\alpha} \otimes u_{\alpha_{1}} \otimes \ldots \otimes u_{\left.\alpha_{n}\right)} .
$$

Os parênteses nos índices subescritos indicam simetrização entre todos os índices contidos entre o parêntesis de abertura e o de fechamento:

$$
F_{\left(\alpha_{1} \alpha_{2} \ldots \alpha_{n}\right)}:=\frac{1}{n !} \sum_{\pi} F_{\alpha_{\pi_{1}} \alpha_{\pi_{2} \ldots \alpha_{\pi_{n}}}}
$$

qualquer que seja a expressão $F$ que dependa de $n$ índices, onde $\left(\pi_{1}, \pi_{2}, \ldots, \pi_{n}\right)$ é uma permutação de $(1,2, \ldots, n)$ e a soma é sobre todas as permutações. Note que

$$
\left(u_{\left(\alpha_{1}\right.} \otimes \ldots \otimes u_{\left.\alpha_{n-1}\right)}, \hat{a}_{\alpha} u_{\left(\beta_{1}\right.} \otimes \ldots \otimes u_{\left.\beta_{n}\right)}\right)=\left(\hat{a}_{\alpha}^{\dagger} u_{\left(\alpha_{1}\right.} \otimes \ldots \otimes u_{\left.\alpha_{n-1}\right)}, u_{\left(\beta_{1}\right.} \otimes \ldots \otimes u_{\left.\beta_{n}\right)}\right),
$$

o que justifica a notação: $\hat{a}_{\alpha}^{\dagger}$ é o adjunto de $\hat{a}_{\alpha}$.

Um operador que será útil mais adiante é o chamado operador número,

$$
\hat{N}:=\int_{\mathcal{J}} d \mu(\alpha) \hat{a}_{\alpha}^{\dagger} \hat{a}_{\alpha}
$$

É facil verificar que sua atuação num elemento arbitrário de $\mathcal{F}_{s}(\mathcal{H})$ é dada, formal- 
mente $\AA^{3}$ por

$$
\hat{N}|\Psi\rangle \equiv \hat{N}\left(\psi_{0}, \psi_{1}, \psi_{2}, \ldots, \psi_{n}, \ldots\right)=\left(0, \psi_{1}, 2 \psi_{2}, \ldots, n \psi_{n}, \ldots\right)
$$

o que justifica seu nome. No entanto, deve-se resistir à tentação de se interpretar $\hat{N}$, num caso genérico, como número de partículas presentes no estado. Apenas em situações muito especiais esse operador pode ser interpretado dessa maneira.

Pela definição de $\hat{a}_{\alpha}$ e $\hat{a}_{\alpha}^{\dagger}$, pode-se verificar que

$$
\left[\hat{a}_{\alpha}, \hat{a}_{\beta}^{\dagger}\right]=\delta(\alpha, \beta)
$$

com todos os outros comutadores se anulando. Essas relações de comutação são chamadas de relações canônicas de comutação (RCC).

Em posse de todos esses elementos, podemos finalmente construir uma representação para os operadores campo e momento canonicamente conjugado,

$$
\begin{aligned}
\hat{\Phi} & :=\int_{\mathcal{J}} d \mu(\alpha)\left(\hat{a}_{\alpha} u_{\alpha}+\hat{a}_{\alpha}^{\dagger} u_{\alpha}^{*}\right), \\
\hat{\Pi} & :=\int_{\mathcal{J}} d \mu(\alpha) \sqrt{{ }^{(3)} g} n^{a}\left(\hat{a}_{\alpha} \nabla_{a} u_{\alpha}+\hat{a}_{\alpha}^{\dagger} \nabla_{a} u_{\alpha}^{*}\right),
\end{aligned}
$$

e, com isso, verificar que estes satisfazem as relações de comutação que desejávamos, Eq. 2.6

Um estado privilegiado dessa representação é o chamado estado de vácuo (da representação),

$$
|0\rangle=(1,0,0, \ldots)
$$

a partir do qual $\mathcal{F}_{s}(\mathcal{H})$ pode ser gerado (pelo fecho do conjunto denso gerado) através de um número finito de aplicações do operador $\hat{a}_{\alpha}^{\dagger}, \alpha \in \mathcal{J}$. Nesse estado, temos, por construção, $\langle\Phi\rangle_{0}=0$ e

$$
\left\langle\Phi(x) \Phi\left(x^{\prime}\right)\right\rangle_{0}=\int_{\mathcal{J}} d \mu(\alpha) u_{\alpha}(x) u_{\alpha}^{*}\left(x^{\prime}\right)
$$

Pode-se mostrar que essa bi-distribuição é simétrica em $\left(x, x^{\prime}\right)$ se a separação de ${ }^{3}$ De modo mais rigoroso, a atuação dada é definida apenas para aqueles elementos de $\mathcal{F}_{s}(\mathcal{H})$ para os quais $\left\|\psi_{1}\right\|^{2}+4\left\|\psi_{2}\right\|^{2}+\ldots+n^{2}\left\|\psi_{n}\right\|^{2}+\ldots<\infty$ 
ambos for tipo-espaço, que é uma implementação da causalidade de Einstein. O mesmo não é verdade se $\left(x, x^{\prime}\right)$ forem causalmente conectados. Essa bi-distribuição possui importância central em TQCEC pois é a partir dela que um dos observáveis mais importante, o valor esperado do tensor energia-momento, é calculado.

Antes de falarmos mais sobre as propriedades de $\left\langle\Phi(x) \Phi\left(x^{\prime}\right)\right\rangle_{0}$ e sua utilidade, devemos fazer uma observação sobre a construção feita acima. A construção descrita está longe de ser única. O ponto de partida, a escolha do espaço $\mathcal{S}_{\mathbb{C}}^{+} \subset \mathcal{S}_{\mathbb{C}}$ gerado por $\left\{u_{\alpha}\right\}_{\alpha \in \mathcal{J}}$ satisfazendo as Eqs. (2.7) e (2.8), pode ser feita de diversas (infinitas!) maneiras diferentes. De fato, dado um conjunto $\left\{u_{\alpha}\right\}_{\alpha \in \mathcal{J}}$ com as propriedades desejadas, podemos construir um outro conjunto $\left\{v_{\alpha^{\prime}}\right\}_{\alpha^{\prime} \in \mathcal{J}^{\prime}}\left(\mathcal{J}^{\prime}\right.$ pode ser diferente de $\mathcal{J})$ com as mesmas propriedades através de

$$
v_{\alpha^{\prime}}:=\int_{\mathcal{J}} d \mu(\alpha)\left(c_{\alpha^{\prime} \alpha} u_{\alpha}+d_{\alpha^{\prime} \alpha} u_{\alpha}^{*}\right)
$$

onde $c_{\alpha^{\prime} \alpha}$ e $d_{\alpha^{\prime} \alpha}$ são constantes complexas satisfazendo

$$
\begin{aligned}
& \int_{\mathcal{J}} d \mu(\alpha)\left(c_{\alpha^{\prime} \alpha}^{*} c_{\beta^{\prime} \alpha}-d_{\alpha^{\prime} \alpha}^{*} d_{\beta^{\prime} \alpha}\right)=\delta\left(\alpha^{\prime}, \beta^{\prime}\right), \\
& \int_{\mathcal{J}} d \mu(\alpha)\left(c_{\alpha^{\prime} \alpha} d_{\beta^{\prime} \alpha}-d_{\alpha^{\prime} \alpha} c_{\beta^{\prime} \alpha}\right)=0 .
\end{aligned}
$$

Como $\left\{v_{\alpha^{\prime}}, v_{\alpha^{\prime}}^{*}\right\}_{\alpha^{\prime} \in \mathcal{J}^{\prime}}$ também tem que ser uma base do espaço $\mathcal{S}_{\mathbb{C}}$, as seguintes condições também devem ser satisfeitas:

$$
\begin{aligned}
& \int_{\mathcal{J}^{\prime}} d \mu\left(\alpha^{\prime}\right)\left(c_{\alpha^{\prime} \alpha} c_{\alpha^{\prime} \beta}^{*}-d_{\alpha^{\prime} \alpha}^{*} d_{\alpha^{\prime} \beta}\right)=\delta(\alpha, \beta), \\
& \int_{\mathcal{J}^{\prime}} d \mu\left(\alpha^{\prime}\right)\left(c_{\alpha^{\prime} \alpha}^{*} d_{\alpha^{\prime} \beta}-d_{\alpha^{\prime} \alpha} c_{\alpha^{\prime} \beta}^{*}\right)=0 .
\end{aligned}
$$

No caso particular em que $c_{\alpha^{\prime} \beta}=c_{\alpha} \delta(\alpha, \beta)$ e $d_{\alpha^{\prime} \beta}=d_{\alpha} \delta(\alpha, \beta)$, com $c_{\alpha}$ e $d_{\alpha}$ constante complexas (e $\left.\mathcal{J}=\mathcal{J}^{\prime}\right)$, então as condições acima são satisfeitas desde que

$$
\left|c_{\alpha}\right|^{2}-\left|d_{\alpha}\right|^{2}=1, \quad \alpha \in \mathcal{J}
$$

Transformações (2.18) (juntamente com sua conjugação complexa) satisfazendo as condições detalhadas acima são chamadas de transformações de Bogoliubov. Elas 
representam não apenas uma mudança de base em $\mathcal{S}_{\mathbb{C}}$ mas também uma escolha diferente de $\mathcal{S}_{\mathbb{C}}^{+}$se, para algum $\alpha^{\prime} \in \mathcal{J}^{\prime}$ e $\alpha \in \mathcal{J}, d_{\alpha^{\prime} \alpha} \neq 0$. Isso, por sua vez, pode levar a uma representação de $\hat{\Phi}$ e $\hat{\Pi}$ sobre um espaço de Fock distinto $\mathcal{F}_{s}(\mathcal{H})$ que não seja unitariamente equivalente à representação anterior.

As transformações de Bogoliubov também podem ser interpretadas como misturando os operadores $\hat{a}_{\alpha}$ e $\hat{a}_{\alpha}^{\dagger}$. Substituindo a decomposição de $u_{\alpha}$ e $u_{\alpha}^{*}$ em termos de $v_{\alpha^{\prime}}$ e $v_{\alpha^{\prime}}^{*}$ na expressão 2.15 e impondo que o resultado seja representado por

$$
\hat{\Phi}=\int_{\mathcal{J}^{\prime}} d \mu\left(\alpha^{\prime}\right)\left(\hat{b}_{\alpha^{\prime}} v_{\alpha^{\prime}}+\hat{b}_{\alpha^{\prime}}^{\dagger} v_{\alpha^{\prime}}^{*}\right)
$$

obtém-se

$$
\begin{aligned}
& \hat{b}_{\alpha^{\prime}}=\int_{\mathcal{J}} d \mu(\alpha)\left(c_{\alpha^{\prime} \alpha}^{*} \hat{a}_{\alpha}-d_{\alpha^{\prime} \alpha}^{*} \hat{a}_{\alpha}^{\dagger}\right), \\
& \hat{b}_{\alpha^{\prime}}^{\dagger}=\int_{\mathcal{J}} d \mu(\alpha)\left(c_{\alpha^{\prime} \alpha} \hat{a}_{\alpha}^{\dagger}-d_{\alpha^{\prime} \alpha} \hat{a}_{\alpha}\right) .
\end{aligned}
$$

Construindo o novo operador número $\hat{N}_{v}:=\int_{\mathcal{J}^{\prime}} d \mu\left(\alpha^{\prime}\right) \hat{b}_{\alpha^{\prime}}^{\dagger} \hat{b}_{\alpha^{\prime}}$, agora associado aos $\operatorname{modos} v_{\alpha^{\prime}}$, seu valor esperado no estado de vácuo associado aos modos $u_{\alpha}$ vale

$$
\left\langle N_{v}\right\rangle_{0}=\int_{\mathcal{J}^{\prime}} d \mu\left(\alpha^{\prime}\right) \int_{\mathcal{J}} d \mu(\alpha)\left|d_{\alpha^{\prime} \alpha}\right|^{2}
$$

\subsection{Valor esperado do tensor energia-momento e estados Hadamard}

Voltemos, agora, para a bi-distribuição $\left\langle\Phi(x) \Phi\left(x^{\prime}\right)\right\rangle_{0}$. A partir dela, o valor esperado do tensor energia-momento no vácuo pode ser calculado por

$$
\begin{aligned}
\left\langle T_{a b}(x)\right\rangle_{0}= & \lim _{x^{\prime} \rightarrow x}\left\{(1-2 \xi) \nabla_{a} \nabla_{b}^{\prime}+\xi R_{a b}-\xi\left(\nabla_{a} \nabla_{b}+\nabla_{a}^{\prime} \nabla_{b}^{\prime}\right)\right. \\
& \left.+(2 \xi-1 / 2) g_{a b}\left[\nabla^{c} \nabla_{c}^{\prime}+\left(m^{2}+\xi R\right)\right]\right\}\left\langle\Phi(x) \Phi\left(x^{\prime}\right)\right\rangle_{0},
\end{aligned}
$$

onde operadores diferenciais com apóstrofo apenas atuam sobre o argumento $x^{\prime}$. Portanto, estamos primordialmente interessados na função de dois pontos $\left\langle\Phi(x) \Phi\left(x^{\prime}\right)\right\rangle_{0}$ 
no limite em que os pontos são feitos coincidirem. No entanto, a expressão 2.17 não é bem definida nesse limite, o que nos obriga a lidar com a renormalização da função de dois pontos se quisermos usá-la para obter qualquer resultado fisicamente aceitável. Esse problema está intimamente relacionado à discussão sobre as flutuações de ponto zero que fizemos na Sec. 1.1. mas agora no contexto mais geral de espaços-tempos curvos.

Tipicamente, há duas possíveis razões para a divergência do limite de coincidência $x^{\prime} \rightarrow x$ na expressão (2.17): o comportamento dos modos $u_{\alpha}$ no regime infra-vermelho (modos com escalas típicas de distância cada vez maiores) e o comportamento no regime ultra-violeta (modos com escalas típicas de distância cada vez menores). Relacionados a esses regimes, dois resultados importantíssimos vêm ao nosso auxílio.

O primeiro deles diz respeito ao regime ultra-violeta. Para uma ampla classe de estados, denominados estados Hadamard, a estrutura da singularidade na função de dois pontos no limite de coincidência independe do estado em particular $\ell$ dessa classe, dependendo apenas de quantidades geométricas locais:

$$
\begin{aligned}
\left\langle\Phi(x) \Phi\left(x^{\prime}\right)\right\rangle_{\ell}= & W_{\ell}\left(x, x^{\prime}\right)+V\left(x, x^{\prime}\right) \ln \left[\sigma^{2}+2 i \epsilon\left(t-t^{\prime}\right)+\epsilon^{2}\right] \\
& +\frac{U\left(x, x^{\prime}\right)}{(2 \pi)^{2}\left[\sigma^{2}+2 i \epsilon\left(t-t^{\prime}\right)+\epsilon^{2}\right]},
\end{aligned}
$$

com

$$
\begin{gathered}
V\left(x, x^{\prime}\right)=\sum_{j=0}^{\infty} v_{j}\left(x, x^{\prime}\right) \sigma^{j}, \\
W_{\ell}\left(x, x^{\prime}\right)=\sum_{j=0}^{\infty} w_{j}^{(\ell)}\left(x, x^{\prime}\right) \sigma^{j},
\end{gathered}
$$

onde $U, v_{j}$ e $w_{j}^{(\ell)}$ são funções suaves, $U(x, x)=1$ e toda dependência no estado $\ell$ encontra-se em $W_{\ell}$. Nessas expressões, $\sigma \equiv \sigma\left(x, x^{\prime}\right)$ é a distância geodésica quadrática entre $x$ e $x^{\prime}$ (sem perda de generalidade, podemos considerar que um ponto se encontra numa vizinhança normal do outro, para que $\sigma$ seja bem definido).

O procedimento de renormalização em espaços curvos consiste, então, em se sub- 
trair da função de dois pontos $\left\langle\Phi(x) \Phi\left(x^{\prime}\right)\right\rangle$ uma bi-distribuição $H\left(x, x^{\prime}\right)$ localmente construída, satisfazendo a equação de Klein-Gordon em ambos os argumentos e com a mesma estrutura de singularidade que $\left\langle\Phi(x) \Phi\left(x^{\prime}\right)\right\rangle$ para $x^{\prime} \rightarrow x$. A escolha de uma tal $H\left(x, x^{\prime}\right)$ que desempenhe esse papel não é única, e isso leva a uma indeterminação no valor exato de $\left\langle\Phi(x) \Phi\left(x^{\prime}\right)\right\rangle$ renormalizado e, portanto, de $\left\langle T_{a b}\right\rangle$. Dentro do escopo de TQCEC, essa indeterminação é inevitável e está relacionada, por exemplo, com a imprevisibilidade do valor da constante cosmológica.

O outro resultado importante diz respeito a outros tipos de singularidade na função de dois pontos, inclusive infra-vermelha. Pode ser mostrado que se um estado é Hadamard, então nenhuma outra singularidade, além da identificada em (2.28), está presente em sua função de dois pontos [12]. Isso garante, por exemplo, que uma vez realizado o procedimento de renormalização numa vizinhança de uma superfície de Cauchy, nenhuma outra divergência poderá aparecer no decurso da evolução do sistema. Ao mesmo tempo que é um resultado tranquilizador, no sentido que não precisamos nos preocupar com divergências adicionais uma vez que efetuamos o procedimento de renormalização, poderia também soar como um resultado desanimador, pois pareceria indicar que se a densidade de energia de vácuo é desprezável num certo instante, então permaneceria sendo irrelevante ao longo de toda a história do sistema. Felizmente, essa última conclusão não é correta e o mecanismo do despertar do vácuo que apresentaremos no próximo capítulo mostra como é possível que, a despeito do bom comportamento da função de dois pontos renormalizada, a densidade de energia do vácuo possa crescer exponencialmente. 


\section{Capítulo 3}

\section{Despertar do vácuo por campos gravitacionais}

Neste capítulo apresentaremos o tema principal desta tese: o efeito do despertar do vácuo ou da dominância do vácuo induzida pela gravitação. Antes de irmos propriamente para o efeito, vamos contextualizá-lo pela motivação que tínhamos quando o descobrimos.

Como vimos nos capítulos anteriores, no paradigma moderno de TQC (seja em espaços curvos ou não), é inevitável que tenhamos que lidar com o fato (verificado experimentalmente através do efeito Casimir) de que mesmo a configuração mais "simples" de um sistema, o vácuo, tem uma estrutura não trivial. No entanto, em espaço plano a densidade de energia desse estado é desprezável na escala macroscópica frente às outras contribuições clássicas, sendo relevante apenas em experimentos cuidadosamente conduzidos para maximizar seus efeitos. Ou seja, embora a teoria quântica tenha desvelado sua rica estrutura, o vácuo continua sendo, no que diz respeito a efeitos macroscópicos observacionais, tão evasivo quanto o completo vazio da Física Clássica.

No entanto, uma vez que a gravidade é considerada, a influência mútua entre a energia de vácuo e a geometria não trivial do espaço-tempo de fundo leva a novas 
possibilidades. Embora a densidade de energia de vácuo seja, usualmente, irrelevante como fonte de gravitação, ela é afetada pela dinâmica do espaço-tempo. Uma questão interessante, então, se coloca: haveria alguma evolução do espaço-tempo de fundo que obrigasse a densidade de energia de vácuo a se tornar importante ou até mesmo dominante na evolução subsequente do sistema? É esperado que em situações extremas, como nas singularidades de buracos negros e, possivelmente, nos primeiros instante do Universo, efeitos quânticos, inclusive da própria gravitação, desempenhem um papel importante. Mas a questão aqui é se haveria geometrias bem comportadas que despertassem todo o potencial do estado de vácuo. Além disso, estamos interessados em efeitos que não advenham do regime ultra-violeta dos campos, pois este está sujeito ao procedimento de renormalização que faz com que, dentro do escopo de TQCEC, sua contribuição seja irremediavelmente indeterminada.

\subsection{Dominância do vácuo induzida pela gravita-}

\section{ção}

Considere um espaço-tempo globalmente hiperbólico cujo passado assintótico seja conformalmente plano e cujo futuro assintótico seja conformalmente estático:

$$
d s^{2} \sim \begin{cases}f_{\text {in }}^{2}\left(-d t^{2}+d \vec{x}^{2}\right), & \text { passado assintótico } \\ f_{\text {out }}^{2}\left(-d t^{2}+h_{i j} d x^{i} d x^{j}\right), & \text { futuro assintótico }\end{cases}
$$

onde $f_{J} \equiv f_{J}(t, \vec{x})>0, J \in\{i n$, out $\}$, são funções suaves e $h_{i j}=h_{i j}(\vec{x})$ são as componentes de uma métrica riemanniana tridimensional arbitrária. Essa forma para o espaço-tempo é considerada para simplificar a análise, não sendo essencial para a ocorrência do efeito. Além disso, há um forte apelo observacional para a forma (3.1) da métrica. Ela modela a formação de objetos astrofísicos estáticos a partir de matéria suficientemente difusa (caso em que $f_{\text {in }}=f_{\text {out }}=1$ ), além de 
modelar a formação de estruturas no nosso Universo em expansão (caso em que $f_{\text {in }}=f_{\text {out }}=a(t)$, o fator de escala da expansão do Universo).

Nesse espaço-tempo, consideremos um campo escalar $\Phi$ regido pela equação de Klein-Gordon 2.2). Nas regiões assintóticas é útil expressar o campo como $\Phi=$ $\widetilde{\Phi} / f_{J}$, de modo que $\widetilde{\Phi}$ satisfaz

$$
-\frac{\partial^{2}}{\partial t^{2}} \widetilde{\Phi}=\left(-\Delta_{J}+V_{J}\right) \widetilde{\Phi}
$$

onde $\Delta_{i n}$ é o laplaciano usual em espaço-plano, $\Delta_{o u t}=h^{i j} D_{i} D_{j}$ é o laplaciano associado à métrica $h_{i j}\left(D_{i}\right.$ é a derivada covariante compatível com $h_{i j}$ ) e o potencial efetivo $V_{J}$ é dado por

$$
\begin{aligned}
V_{J} & =\frac{\left(\Delta_{J} f_{J}-\ddot{f}_{J}\right)}{f_{J}}+f_{J}^{2}\left(m^{2}+\xi R\right) \\
& =(1-6 \xi) \frac{\left(\Delta_{J} f_{J}-\ddot{f}_{J}\right)}{f_{J}}+f_{J}^{2} m^{2}+\xi K_{J},
\end{aligned}
$$

onde $K_{\text {in }} \equiv 0$ e $K_{\text {out }}$ é o escalar de curvatura associado à métrica $h_{i j}$.

Nos casos em que $V_{J}=V_{J}(\vec{x})$, a Eq. (3.2) pode ser resolvida analisando-se o problema de auto-valores do operador $\left(-\Delta_{J}+V_{J}\right)$ após o procedimento de separação de variáveis. Em analogia com equações como a de Schrödinger, sabemos que embora operadores do tipo $-\Delta_{J}$ sejam positivos, um potencial suficientemente negativo $V_{J}$ pode fazer com que auto-valores negativos contribuam para o espectro de $\left(-\Delta_{J}+\right.$ $\left.V_{J}\right)$. Isso, por sua vez, faria com que soluções com comportamento exponencial real necessariamente contribuissem para gerar soluções arbitrárias de (3.2). Em outras palavras, se $V_{J}=V_{J}(\vec{x})$ for suficientemente negativo em alguma região, então qualquer base do espaço $S_{\mathbb{C}}$ e também de $S_{\mathbb{C}}^{+}$conterá elementos com comportamento exponencial real na variável $t$.

Para tornar a discussão ainda mais explícita, suponhamos que $f_{\text {in }}=f_{\text {out }}=1$ (espaço-tempo assintoticamente plano no passado e estático no futuro), como será útil em nossa aplicação astrofísica, e ainda $m=0$. Nesse caso, $V_{\text {in }}=0$ e $V_{\text {out }}=\xi R$. Então, uma possível base $\left\{u_{\vec{k}}, u_{\vec{k}}^{*}\right\}_{\vec{k} \in \mathbb{R}^{3}}$ para $S_{\mathbb{C}}$ é aquela constituída de soluções da 
equação de Klein-Gordon em todo o espaço-tempo que assintoticamente no passado (e só lá) assumem a forma de ondas planas:

$$
u_{\vec{k}} \stackrel{\text { passado }}{\sim} \frac{e^{-i(\omega t-\vec{k} \cdot \vec{x})}}{\sqrt{16 \pi^{3} \omega}}
$$

onde $\omega:=\|\vec{k}\|$. A conveniência de se escolher essa base é porque os modos $\left\{u_{\vec{k}}\right\}_{\vec{k} \in \mathbb{R}^{3}}$ selecionam o subespaço $S_{\mathbb{C}}^{+}$que leva, pela construção do capítulo anterior, ao espaço de Fock de representação para $\hat{\Phi}$ cujo estado de vácuo, $|0\rangle_{i n}$, é exatamente o estado de vácuo usual de TQC em espaço plano. Ou seja, para observadores inerciais no passado assintótico, o estado de vácuo dessa representação representa ausência total de partículas do campo $\hat{\Phi}$. Além disso, após renormalização, a densidade de energia desse vácuo, nessa região, é nula, por definição. Adotaremos, então, esse como sendo o estado do campo, modelando uma situação que inicialmente é a mais simples possível e analisando a evolução subsequente.

Notemos que uma vez fixado o estado do campo, no caso o vácuo $|0\rangle_{\text {in }}$ da representação associada a $\left\{u_{\vec{k}}\right\}_{\vec{k} \in \mathbb{R}^{3}}$, o campo permanecerá nesse estado durante toda a história do espaço-tempo. Assim, passado o período transiente que levou a geometria da configuração plana no passado para a estática mas curva no futuro, o campo continuará no estado de vácuo da representação associada a $\left\{u_{\vec{k}}\right\}_{\vec{k} \in \mathbb{R}^{3}}$, embora agora, no futuro, esses modos não se pareçam em nada, em geral, com soluções estacionárias.

Sejam $\left\{v_{\alpha}, v_{\alpha}^{*}\right\}_{\alpha \in \mathcal{J}}$ as soluções da equação de Klein-Gordon que no futuro assintótico assumem a forma estacionária:

$$
v_{\alpha} \stackrel{\text { futuro }}{\sim} \frac{e^{-i \varpi_{\alpha} t}}{\sqrt{2 \varpi_{\alpha}}} F_{\alpha}(\vec{x})
$$

onde $\varpi_{\alpha}>0$ e $F_{\alpha}$ são soluções do problema de auto-valor/auto-função

$$
\left[-\Delta_{\text {out }}+V_{\text {out }}(\vec{x})\right] F_{\alpha}(\vec{x})=\varpi_{\alpha}^{2} F_{\alpha}(\vec{x})
$$


satisfazendo, ainda, a condição de normalização

$$
\int_{\Sigma_{\text {out }}} d^{3} x \sqrt{h} F_{\alpha}(\vec{x})^{*} F_{\beta}(\vec{x})=\delta(\alpha, \beta)
$$

onde $\Sigma_{\text {out }}$ é uma superfície de Cauchy no futuro assintótico. [Essa condição de normalização vem da condição (2.7) aplicada a (3.5).] Esses são os modos que definem, no futuro, a representação cujo estado de vácuo, $|0\rangle_{\text {out }}$, representa a ausência de partículas do campo $\hat{\Phi}$. O fato que, em geral, a relação entre as bases $\left\{u_{\vec{k}}, u_{\vec{k}}^{*}\right\}_{\vec{k} \in \mathbb{R}^{3}}$ e $\left\{v_{\alpha}, v_{\alpha}^{*}\right\}_{\alpha \in \mathcal{J}}$ envolve uma transformação de Bogoliubov em que pelo menos algum $d_{\vec{k} \alpha}$ seja não nulo implica que o estado $|0\rangle_{\text {in }}$ contém partículas segundo observadores estáticos no futuro assintótico. Esse é o fenômeno de criação de partículas por campos gravitacionais, que está na base do efeito Hawking e da criação de partículas em universos em expansão.

Nosso interesse, no entanto, é outro [13]. Se a Eq. (3.6) admitir um (e apenas um, para simplificar a argumentação) auto-valor negativo, digamos $-\Omega^{2}, \operatorname{com} \Omega>0$, então o conjunto $\left\{v_{\alpha}, v_{\alpha}^{*}\right\}_{\alpha \in \mathcal{J}}$ deixará de ser uma base de $\mathcal{S}_{\mathbb{C}}$, pois deixará de ser completo. Para completá-lo devemos incluir a solução da equação de Klein-Gordon que no futuro toma a forma

$$
w_{\Omega} \stackrel{\text { futuro }}{\sim} e^{i \delta} \frac{\left(e^{\Omega t-i \theta}+e^{-\Omega t+i \theta}\right)}{\sqrt{4 \Omega \sin (2 \theta)}} \bar{F}_{\Omega}(\vec{x})
$$

e seu conjugado complexo, onde $0<\theta \leq \frac{\pi}{4}$, $\delta$ é uma fase arbitrária e

$$
\begin{aligned}
& \int_{\Sigma_{\text {out }}} d^{3} x \sqrt{h} \bar{F}_{\Omega}(\vec{x})^{*} \bar{F}_{\Omega}(\vec{x})=1, \\
& \int_{\Sigma_{\text {out }}} d^{3} x \sqrt{h} \bar{F}_{\Omega}(\vec{x})^{*} F_{\alpha}(\vec{x})=0 .
\end{aligned}
$$

Note que a condição de normalização 2.7 impede que façamos uso apenas da exponencial decrescente $e^{-\Omega t}$. Isso significa, devido a completeza de ambos os conjuntos $\left\{u_{\vec{k}}, u_{\vec{k}}^{*}\right\}_{\vec{k} \in \mathbb{R}^{3}}$ e $\left\{w_{\Omega}, v_{\alpha}, w_{\Omega}^{*}, v_{\alpha}^{*}\right\}_{\alpha \in \mathcal{J}}$, que pelo menos algum dos modos $u_{\vec{k}}$, que tem a forma de onda plana no passado assintótico, sofrerá um crescimento exponencial assintoticamente no futuro, independentemente de a transformação de Bogoliubov 
entre $\left\{u_{\vec{k}}, u_{\vec{k}}^{*}\right\}_{\vec{k} \in \mathbb{R}^{3}}$ e $\left\{w_{\Omega}, v_{\alpha}, w_{\Omega}^{*}, v_{\alpha}^{*}\right\}_{\alpha \in \mathcal{J}}$ envolver ou não coeficientes $d_{\vec{k} \alpha} \neq 0$ ou $d_{\vec{k} \Omega} \neq 0$. Isso pode ser visto da condição 2.21 aplicada a este caso:

$$
\int_{\mathbb{R}^{3}} d^{3} k\left(\left|c_{\vec{k} \Omega}\right|^{2}-\left|d_{\vec{k} \Omega}\right|^{2}\right)=1
$$

$\operatorname{logo}, c_{\vec{k} \Omega} \neq 0$ para algum $\vec{k}$. Denotando por $\vec{k}^{\prime}$ os valores de $\vec{k}$ para os quais $c_{\vec{k} \Omega} \neq 0$, temos

$$
u_{\vec{k}^{\prime}} \stackrel{\text { futuro }}{\sim}\left(c_{\vec{k}^{\prime} \Omega}+d_{\vec{k}^{\prime} \Omega}\right) \frac{e^{\Omega t} \bar{F}_{\Omega}(\vec{x})}{\sqrt{4 \Omega \sin (2 \theta)}}
$$

onde, por simplicidade, adotamos $\delta=\theta$ e escolhemos, sem perda de generalidade, $\bar{F}_{\Omega}$ real. Em outras palavras, mesmo que a transição entre as geometrias assintóticas se dê de maneira suficientemente "adiabática" para que não haja criação de partículas (apreciável), ainda assim o crescimento exponencial de modos que inicialmente eram ondas planas é inevitável. A consequência física disso é evidente quando recordamos a Eq. (2.17): esse crescimento exponencial leva a uma amplificação das flutuações de vácuo,

$$
\begin{aligned}
\left\langle\Phi(x) \Phi\left(x^{\prime}\right)\right\rangle_{0} & =\int_{\mathbb{R}^{3}} d^{3} k u_{\vec{k}}(x) u_{\vec{k}}^{*}\left(x^{\prime}\right) \\
& \stackrel{\text { futuro }}{\sim} \frac{e^{\Omega\left(t+t^{\prime}\right)} \bar{F}_{\Omega}(\vec{x}) \bar{F}_{\Omega}\left(\vec{x}^{\prime}\right)}{4 \Omega \sin (2 \theta)} \int_{\mathbb{R}^{3}} d^{3} k\left(\left|c_{\vec{k} \Omega}\right|^{2}+\left|d_{\vec{k} \Omega}\right|^{2}+c_{\vec{k} \Omega} d_{\vec{k} \Omega}^{*}+c_{\vec{k} \Omega}^{*} d_{\vec{k} \Omega}\right) \\
& =\frac{e^{\Omega\left(t+t^{\prime}\right)} \bar{F}_{\Omega}(\vec{x}) \bar{F}_{\Omega}\left(\vec{x}^{\prime}\right)}{4 \Omega \sin (2 \theta)}\left[1+2 \int_{\mathbb{R}^{3}} d^{3} k\left(\left|d_{\vec{k} \Omega}\right|^{2}+c_{\vec{k} \Omega} d_{\vec{k} \Omega}^{*}\right)\right] \\
& =: \frac{\kappa e^{\Omega\left(t+t^{\prime}\right)} \bar{F}_{\Omega}(\vec{x}) \bar{F}_{\Omega}\left(\vec{x}^{\prime}\right)}{4 \Omega \sin (2 \theta)},
\end{aligned}
$$

onde $\kappa \sim 1$ é uma constante adimensional (definida pela última igualdade acima) cujo valor exato depende dos detalhes da evolução do espaço-tempo. Na expressão acima estamos considerando que o estado em questão é Hadamard, de modo que o procedimento de renormalização pode ser levado a cabo, não afetando a contribuição dominante do modo $w_{\Omega}$ que nada tem a ver com o regime ultra-violeta. Substituindo essa função de dois pontos na expressão para o valor esperado do tensor energia- 
momento, Eq. (2.27), temos

$$
\begin{gathered}
\left\langle T_{00}\right\rangle \stackrel{\text { futuro }}{\sim}\left\langle\Phi^{2}\right\rangle\left\{\frac{(1-4 \xi)}{2}\left(\Omega^{2}+\frac{\left(D \bar{F}_{\Omega}\right)^{2}}{\bar{F}_{\Omega}^{2}}+\xi K_{\text {out }}\right)+\mathcal{O}\left(e^{-\epsilon t}\right)\right\}, \\
\left\langle T_{0 i}\right\rangle \stackrel{\text { futuro }}{\sim}\left\langle\Phi^{2}\right\rangle\left\{(1-4 \xi) \frac{\Omega D_{i} \bar{F}_{\Omega}}{\bar{F}_{\Omega}}+\mathcal{O}\left(e^{-\epsilon t}\right)\right\}, \\
\left\langle T_{i j}\right\rangle \stackrel{\text { futuro }}{\sim}\left\langle\Phi^{2}\right\rangle\left\{(1-2 \xi) \frac{D_{i} \bar{F}_{\Omega} D_{j} \bar{F}_{\Omega}}{\bar{F}_{\Omega}^{2}}-2 \xi \frac{D_{i} D_{j} \bar{F}_{\Omega}}{\bar{F}_{\Omega}}+\xi \widetilde{R}_{i j}\right. \\
\left.+\frac{(1-4 \xi) h_{i j}}{2}\left(\Omega^{2}-\frac{\left(D \bar{F}_{\Omega}\right)^{2}}{\bar{F}_{\Omega}^{2}}-\xi K_{\text {out }}\right)+\mathcal{O}\left(e^{-\epsilon t}\right)\right\},
\end{gathered}
$$

onde $\epsilon>0, \widetilde{R}_{i j}$ é o tensor de Ricci associado a $h_{i j}$ e

$$
\left\langle\Phi^{2}\right\rangle:=\lim _{x^{\prime} \rightarrow x}\left\langle\Phi(x) \Phi\left(x^{\prime}\right)\right\rangle_{0} \stackrel{\text { futuro }}{\sim} \frac{\kappa e^{2 \Omega t} \bar{F}_{\Omega}(\vec{x})^{2}}{4 \Omega \sin (2 \theta)}
$$

Temos, então, o seguinte cenário: a densidade de energia de vácuo do campo quântico $\hat{\Phi}$, que é nula ou desprezável inicialmente, pode ser exponencialmente amplificada pela evolução bem comportada do espaço-tempo de fundo, dependendo, apenas, se a geometria final é tal que o operador $\left[-\Delta_{\text {out }}+V_{\text {out }}(\vec{x})\right]$ possui autovalores negativos. A viabilidade disso é inquestionável, uma vez que $V_{\text {out }}$ depende do acoplamento $\xi$ e, portanto, pode ser feito tão negativo quanto se queira. A questão é se existiria um campo gravitacional realista que seria capaz de levar a um auto-valor negativo mesmo para valores mais "naturais" $|\xi| \sim 1$. Para identificar situações promissoras, façamos uma estimativa. Para um campo sem massa com acoplamento $\xi$ da ordem de $1, V_{\text {out }}$ é da ordem de grandeza do escalar de curvatura $R$. Este, por sua vez, de acordo com a equação de Einstein, é da ordem de grandeza de $8 \pi G \rho_{c}$, onde $\rho_{c}$ é a densidade de energia clássica responsável pela geometria de fundo. Além disso, podemos manipular o sinal de $V_{\text {out }}$ através de valores de $\xi$ (mas ainda mantendo $|\xi| \sim 1$ ). Esses fatos juntos, unidos a analogias com a equação de Schrödinger com potenciais, sugerem que busquemos por cenários em que a variação da densidade de energia clássica, $\delta \rho_{c}$ se dê em distâncias típicas $L$ tais que 
(recuperando unidades)

$$
\frac{8 \pi G \delta \rho_{c} L^{2}}{c^{2}} \approx\left(\frac{\delta \rho_{c} / c^{2}}{10^{15} \mathrm{~g} / \mathrm{cm}^{3}}\right)\left(\frac{L}{7 \mathrm{~km}}\right)^{2} \approx\left(\frac{\delta \rho_{c} / c^{2}}{\rho_{m 0}}\right)\left(\frac{L}{4,7 \mathrm{Gpc}}\right)^{2} \sim 1
$$

onde $\rho_{m 0} \approx 2,5 \times 10^{-30} \mathrm{~g} / \mathrm{cm}^{3}$ é a densidade de matéria média, em escalas cosmológicas, no Universo atual. Em resumo, cenários em que haja uma variação de densidade clássica da ordem de $10^{15} \mathrm{~g} / \mathrm{cm}^{3}$ em escalas de $10 \mathrm{~km}$ ou da ordem de $10^{-30} \mathrm{~g} / \mathrm{cm}^{3} \mathrm{em}$ escalas de 5 Gpc são promissores para que a amplificação exponencial da densidade de energia de vácuo seja desencadeada.

Uma vez disparado o efeito, o valor de $\Omega^{-1}$ fornece a escala de tempo do crescimento exponencial. Tipicamente, $\Omega^{2} \sim\left|V_{\text {out }}\right| \sim 8 \pi G \rho_{c}$. Combinando isso com a estimativa (3.18), temos que $\Omega^{-1} \sim L$. Ou seja, a escala de tempo do crescimento exponencial, quando ocorre, é dada pelo tempo que a luz demora para percorrer a escala de distância $L$. Com isso, vemos que se o vácuo for "despertado" em contextos astrofísicos, a dominância de sua densidade de energia poderia se dar num intervalo de tempo bastante curto, enquanto que num possível cenário cosmológico o crescimento exponencial se daria em uma escala de tempo de bilhões de anos.

A seguir, exploraremos em mais detalhe a possibilidade do despertar do vácuo no contexto astrofísico de objetos compactos.

\subsection{Despertando o vácuo em estrelas relativísticas}

Nesta seção analisaremos em mais detalhe a possibilidade de a densidade de energia de vácuo de um campo $\hat{\Phi}$ sofrer uma amplificação exponencial num contexto astrofísico específico [14]. Pela estimativa (3.18) podemos antecipar que campos gravitacionais associados a objetos compactos, como estrelas de nêutrons, são promissores para desencadear o "despertar do vácuo". Isso porque $10^{14-17} \mathrm{~g} / \mathrm{cm}^{3}$ são densidades típicas de estrelas de nêutrons, cujo valor típico de raio é próximo a $10 \mathrm{~km}$. 
Novamente, consideremos um espaço-tempo com duas regiões assintóticas: plana no passado e esfericamente simétrica e estática no futuro. Com isso queremos modelar a situação de um objeto compacto esférico formado a partir de matéria que, inicialmente, encontrava-se bastante difusa:

$$
d s^{2} \sim \begin{cases}-d t^{2}+d \vec{x}^{2}, & \text { passado } \\ f\left(-d t^{2}+d \chi^{2}\right)+r^{2}\left(d \theta^{2}+\sin \theta^{2} d \varphi^{2}\right), & \text { futuro }\end{cases}
$$

onde $f=f(\chi)>0$ e $r=r(\chi) \geq 0$ são funções apenas da coordenada $\chi$, com $f(\chi) \rightarrow$ 1 e $r(\chi) / \chi \rightarrow 1$ para $\chi \rightarrow \infty$, e $d r / d \chi>0$ de modo que não haja uma superfície tipoluz aprisionada (o que levaria, inevitavelmente, à formação de uma singularidade). Consideremos, ainda, o campo $\hat{\Phi}$ em seu estado de vácuo natural $|0\rangle_{\text {in }}$ no passado assintótico, associado à representação selecionada pelos modos (3.4).

No futuro assintótico, os modos estacionários que definem o vácuo $|0\rangle_{\text {out }}$ são dados por

$$
v_{\varpi l \mu} \stackrel{\text { futuro }}{\sim} \frac{e^{-i \varpi t}}{\sqrt{2 \varpi} f(\chi)} \frac{F_{\varpi l}(\chi)}{r(\chi)} Y_{l \mu}(\theta, \varphi)
$$

onde $Y_{l \mu}(l=0,1,2, \ldots$ e $\mu=-l, \ldots, l)$ são os harmônicos esféricos usuais e $F_{\varpi l}$ são soluções da equação

$$
\left(-\frac{d^{2}}{d \chi^{2}}+V_{\mathrm{ef}}^{(l)}\right) F_{\varpi l}=\varpi^{2} F_{\varpi l}
$$

sujeitas a condições de contorno apropriadas e normalização. O potencial efetivo $V_{\mathrm{ef}}^{(l)}=V_{\mathrm{ef}}^{(l)}(\chi)$ é dado por

$$
V_{\mathrm{ef}}^{(l)}=f\left(m^{2}+\xi R+\frac{l(l+1)}{r^{2}}\right)+\frac{1}{r} \frac{d^{2} r}{d \chi^{2}} .
$$

Utilizando a equação de Einstein, podemos expressar o potencial efetivo acima diretamente em termos da distribuição de matéria responsável pela curvatura do espaçotempo. Supondo, por exemplo, que o objeto compacto seja constituído de fluido perfeito, o potencial efetivo pode ser rescrito como

$$
V_{\mathrm{ef}}^{(l)}=f\left[m^{2}+(\xi-1 / 6) R+\frac{l(l+1)}{r^{2}}+\frac{8 \pi G}{3}(\bar{\rho}-\rho)\right],
$$


onde $\rho=\rho(\chi)$ é a densidade de matéria/energia do objeto compacto e $\bar{\rho}=\bar{\rho}(\chi)$ é a média da densidade desde o centro do objeto até a coordenada $\chi: \bar{\rho}(\chi):=$ $3 M(\chi) /\left(4 \pi r(\chi)^{3}\right)$, com $M(\chi)$ sendo a massa do objeto até a coordenada $\chi$. O escalar de curvatura também pode ser rescrito como $R=8 \pi G(\rho-3 p)$, onde $p$ é a pressão que sustenta a configuração do objeto compacto.

Como explicado na seção anterior, devemos investigar a possibilidade de o operador $\left(-d^{2} / d \chi^{2}+V_{\mathrm{ef}}^{(l)}\right)$ possuir auto-valores negativos:

$$
\left(-\frac{d^{2}}{d \chi^{2}}+V_{\mathrm{ef}}^{(l)}\right) \bar{F}_{\Omega l}=-\Omega^{2} \bar{F}_{\Omega l}
$$

Em caso afimativo, o conjunto $\left\{v_{\varpi l \mu}, v_{\varpi l \mu}^{*}\right\}$ deve ser suplementado com modos

$$
w_{\Omega l \mu} \stackrel{\text { futuro }}{\sim} \frac{\left(e^{-\Omega t+i \pi / 12}+e^{\Omega t-i \pi / 12}\right)}{\sqrt{2 \Omega}} \frac{\bar{F}_{\Omega l}(\chi)}{r(\chi)} Y_{l \mu}(\theta, \varphi)
$$

e seu conjugado complexo para garantir a completeza da base. E isso, por sua vez, levaria ao crescimento exponencial das flutuações de vácuo, acarretando o crescimento exponencial de sua densidade de energia. Mas para que isso ocorra, $V_{\text {ef }}^{(l)}$ deve ser suficientemente negativo numa região suficientemente extensa. Daí vemos que o operador associado ao modo esfericamente simétrico $(l=0)$ de campos sem massa $(m=0)$ é o mais favorável à existência de auto-valor negativo.

A Fig. 3.1 abaixo apresenta, num diagrama de acoplamento $\xi$ e razão massaraio $M / R_{s}$ do objeto compacto, o resultado da busca (numérica) por auto-valores negativos do operador $\left(-d^{2} / d \chi^{2}+V_{\mathrm{ef}}^{(0)}\right)$ para $m=0$ e considerando, por simplicidade, o objeto compacto com densidade uniforme. A Fig. 3.2 apresenta o mesmo tipo de diagrama mas agora para o caso de um objeto compacto com perfil parabólico de densidade, $\rho=\rho_{c}\left(1-r^{2} / R_{s}^{2}\right)$, que é mais próximo à realidade [15], onde $\rho_{c}=$ $15 M /\left(8 \pi R_{s}^{3}\right)$ é densidade central do objeto. Vemos, então, que o campo gravitacional bem comportado de alguns objetos compactos pode, de fato, desencadear o fenômeno do despertar do vácuo desde que exista na Natureza um campo com o acoplamento $\xi$ apropriado. Utilizando a Eq. (3.14) e recuperando as unidades apropriadas, a 


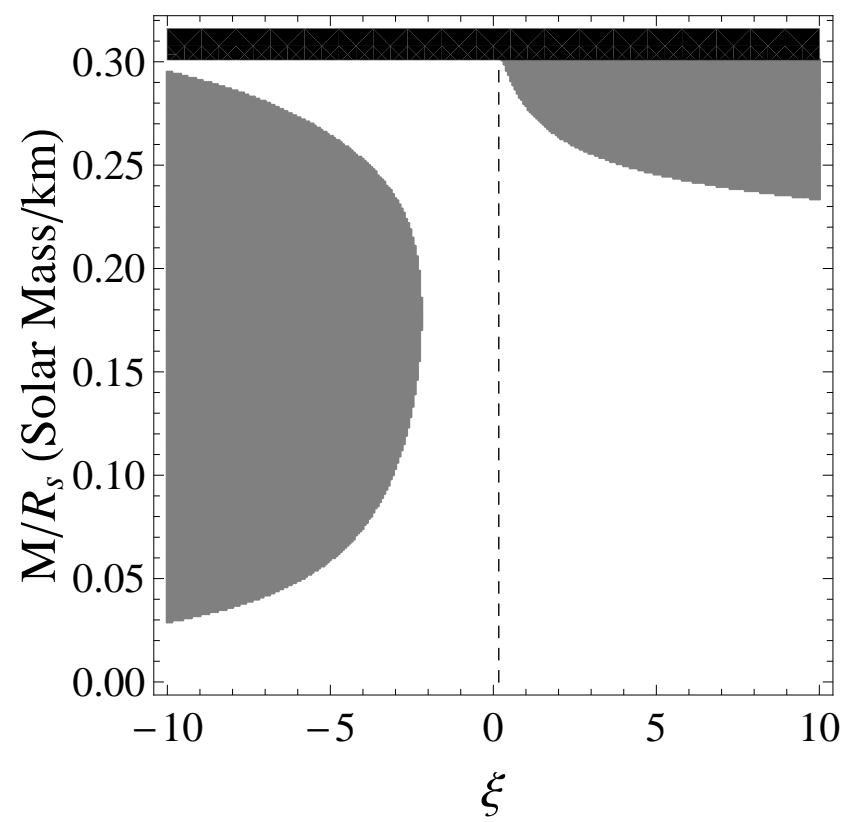

Figura 3.1 - Diagrama mostrando os valores de razão massa-raio de um objeto compacto, com densidade uniforme, para os quais a densidade da energia de vácuo do campo sem massa $\hat{\Phi}$ com acomplamento $\xi$ sofre uma amplificação exponencial (região cinza-escuro). A linha tracejada vertical representa o valor de acoplamento conforme $\xi=1 / 6$ e a região negra representa os valores de razão massa-raio para os quais não há configuração clássica de equilíbrio para o objeto compacto com densidade uniforme.

contribuição dominante da densidade de energia de vácuo assintoticamente no futuro seria dada por

$$
\begin{aligned}
\rho_{V} \stackrel{\text { futuro }}{ } & \frac{\Omega}{R_{s}^{3}} h(\bar{r}) e^{2 \Omega t} \\
& \sim h(\bar{r}) \exp \left(\frac{t /\left(10^{-5} \mathrm{~s}\right)}{R_{s} /(10 \mathrm{~km})}\right) \frac{10^{-62} \mathrm{~g} / \mathrm{cm}^{3}}{R_{s}^{4} /(10 \mathrm{~km})^{4}}
\end{aligned}
$$

onde $h(\bar{r})$ é uma função adimensional de $\bar{r}:=r / R_{s}$ cuja forma exata depende dos detalhes da evolução do espaço-tempo mas que tipicamente assume valores $|h(\bar{r})| \sim 1$ para $\bar{r} \lesssim 1$ e decresce exponencialmente para $\bar{r} \rightarrow \infty$. Além disso, usamos na expressão acima que $\Omega \sim R_{s}^{-1}$, como já discutido anteriormente. Dessa expressão vemos que uma vez despertado o crescimento exponencial, poucos milissegundos são suficientes para fazer a densidade de energia do vácuo sair de seus ínfimos valores típicos, nesse caso da ordem de $1 / R_{s}^{4} \sim 10^{-62} \mathrm{~g} / \mathrm{cm}^{3}$, e atingir valores comparáveis à densidade da própria estrela de nêutrons, $10^{15-17} \mathrm{~g} / \mathrm{cm}^{3}$. Evidentemente, nesse ponto a retro-ação da energia de vácuo sobre o espaço-tempo de fundo se torna inevitável. O efeito dessa retro-ação deve levar o sistema a uma nova configuração 


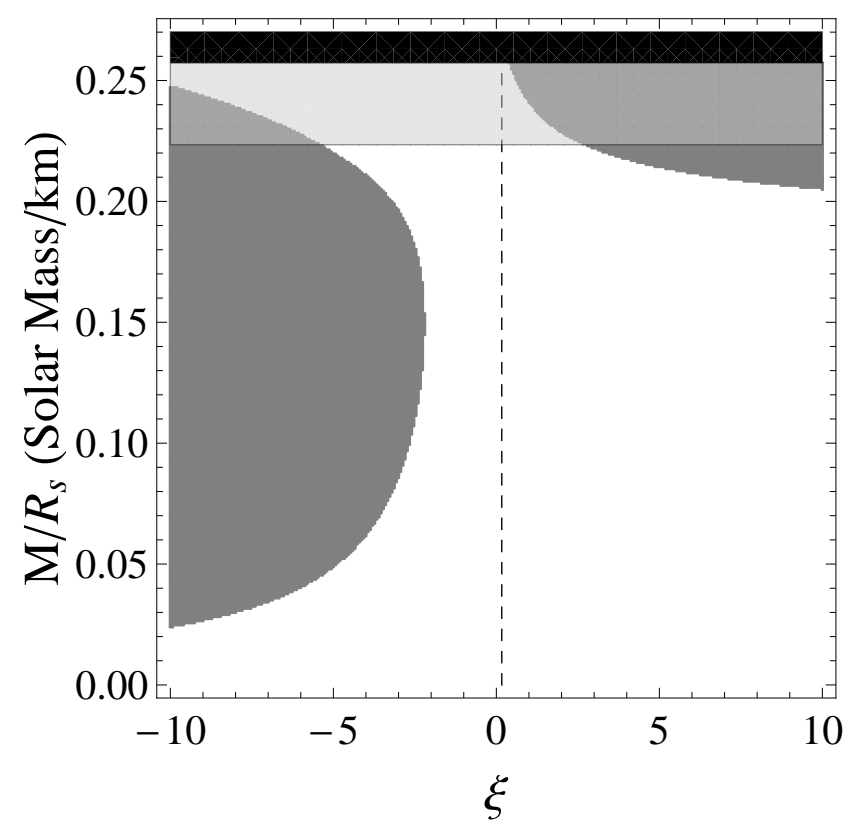

Figura 3.2 - Diagrama mostrando os valores de razão massa-raio de um objeto compacto, com perfil parabólico de densidade, para os quais a densidade da energia de vácuo do campo sem massa $\hat{\Phi}$ com acomplamento $\xi$ sofre uma amplificação exponencial (região cinza-escuro). A linha tracejada vertical representa o valor de acoplamento conforme $\xi=1 / 6$ e a região negra representa os valores de razão massa-raio para os quais não há configuração clássica de equilíbrio para o objeto compacto. A região cinza-claro representa valores de razão massa-raio para os quais as configurações clássicas de equilíbrio do objeto compacto são instáveis para a maioria das equações de estado realistas (veja, e.g., Ref. [15]).

de equilíbrio, cessando, então, o crescimento ilimitado de $\rho_{V}$. E nesse processo de restabilização, outro fenômeno interessante é desencadeado.

Antes de discutirmos a restabilização desse sistema, vale mencionar alguns pontos. O primeiro é que, a despeito do crescimento exponencial da densidade de energia de vácuo, a energia de vácuo total é mantida constante, pelo menos enquanto a sua retro-ação puder ser desprezada e o espaço-tempo se mantiver (aproximadamente) estático. Isso é uma consequência direta, num espaço-tempo estático de fundo, da conservação covariante do valor esperado do tensor energia-momento, $\nabla_{\mu}\left\langle T^{\mu \nu}\right\rangle=0$, e matematicamente por ser mostrado explicitamente pelo fato de a função $h(\bar{r})$ possuir integral volumétrica nula.

Outro ponto é a existência ou não de um campo $\Phi$ com acoplamento $\xi$ apropriado para que seu vácuo seja despertado. Certamente, tal campo não encontra lugar no Modelo Padrão das Partículas Elementares. No entanto, devemos lembrar que as partículas/campos desse modelo dão conta de apenas $5 \%$ da densidade de energia 
total presente em nosso Universo, havendo muito espaço, então, para campos cuja existência ainda desconhecemos. Além disso, o despertar do vácuo fornece uma ligação interessante entre TQCEC e astrofísica observacional, podendo ser usado para se descartar a existência de campos que só interagiriam conosco via gravitação, pois a observação de um objeto compacto estável com um dado valor de razão massa-raio excluiria a possibilidade de existir campos com acoplamento $\xi$ que teriam seus vácuos despertados por esse objeto. Num universo em que $95 \%$ do conteúdo energético é desconhecido, uma maneira observacional de se descartar a existência de campo livres é bem-vinda.

\subsection{Restabilização e produção de partículas}

Como mencionado acima, uma vez que a densidade de energia de vácuo atinge valores comparáveis à das componentes clássicas, sua retro-ação sobre o espaçotempo de fundo é inevitável e é razoável esperar que o sistema atinja uma nova configuração de equilíbrio. Consideraremos, aqui, um cenário no qual após um período de crescimento exponencial da densidade de energia de vácuo, no qual o espaço-tempo é aproximadamente estático (região B na expressão abaixo), o sistema é levado a uma nova configuração de equilíbrio, na qual o crescimento exponencial das flutuações de vácuo cessa. Essa nova fase estável será representada pela região C na expressão abaixo, que complementa a situação descrita por (3.19):

$$
d s^{2}=\left\{\begin{array}{ll}
-d t^{2}+d \vec{x}^{2} & (\mathrm{~A}) \\
N_{(\mathrm{B})}^{2}\left(-d t^{2}+h_{i j}^{(\mathrm{B})} d x^{i} d x^{j}\right) & (\mathrm{B}) \\
N_{(\mathrm{C})}^{2}\left(-d t^{2}+h_{i j}^{(\mathrm{C})} d x^{i} d x^{j}\right) & (\mathrm{C})
\end{array} .\right.
$$

Aqui, $N_{(J)}=N_{(J)}(\vec{x})>0, J \in\{\mathrm{B}, \mathrm{C}\}$, são funções suaves e $h_{i j}^{(J)}=h_{i j}^{(J)}(\vec{x})$. Como, por hipótese, a região C não possui modos com crescimento exponencial, podemos escrever $\hat{\Phi}$ como

$$
\hat{\Phi}=\int d \mu(\alpha)\left[\hat{b}_{\alpha} \nu_{\alpha}+\hat{b}_{\alpha}^{\dagger} \nu_{\alpha}^{*}\right]
$$


onde $\nu_{\alpha}$ são os modos normais que na região $C$ tomam a forma

$$
\nu_{\alpha} \stackrel{(\mathrm{C})}{=} \frac{e^{-i \varpi_{\alpha} t}}{\sqrt{2 \varpi_{\alpha}} N_{(\mathrm{C})}(\vec{x})} F_{\alpha}^{(\mathrm{C})}(\vec{x}), \quad \varpi_{\alpha}>0
$$

Além disso, como a região C é estática, podemos perguntar qual será o conteúdo de partículas do campo $\hat{\Phi}$ nessa região no estado $|0\rangle_{i n}$. O ponto principal aqui será verificarmos que as flutuações do estado de vácuo $|0\rangle_{i n}$, amplificadas exponencialmente durante a fase B, não podem ser acomodadas como meras flutuações do estado de vácuo natural na região $\mathrm{C},|0\rangle_{\text {out }}$. Como consequência disso, a restabilização do sistema levará, inevitavelmente, à criação de partículas do campo $\hat{\Phi}$, mesmo no limite em que a variação da geometria de fundo seja suficientemente lenta 16. Isso distingue esse efeito de criação de partículas daquele devido à variação do espaço-tempo, responsável pela evaporação de buracos negros [1].

Para estimar o número esperado de partículas criadas, consideraremos uma situação simplificada em que o espaço-tempo é simétrico por reversão temporal em relação a uma superfície de Cauchy $\Sigma_{t_{S}}$ contida na região B. Nesse caso, a Eq. (3.27) toma a forma

$$
d s^{2}= \begin{cases}-d t^{2}+d \vec{x}^{2} & (\mathrm{~A}) \\ N_{(\mathrm{B})}^{2}\left(-d t^{2}+h_{i j}^{(\mathrm{B})} d x^{i} d x^{j}\right) & (\mathrm{B}) \\ -d t^{2}+d \vec{x}^{2} & (\mathrm{C})\end{cases}
$$

Os modos $u_{\vec{k}}$ e $\nu_{\vec{k}}$ que selecionam as representações cujo estado de vácuo representa ausência de partículas em cada uma das regiões A e C, respectivamente, são dados por

$$
\begin{gathered}
u_{\vec{k}} \stackrel{(\mathrm{A})}{=}\left(16 \pi^{3} \omega\right)^{-1 / 2} \exp [-i(\omega t-\vec{k} \cdot \vec{x})] \\
\nu_{\vec{k}} \stackrel{(\mathrm{C})}{=}\left(16 \pi^{3} \omega\right)^{-1 / 2} \exp [-i(\omega t-\vec{k} \cdot \vec{x})]
\end{gathered}
$$

Para analisarmos o conteúdo de partículas do estado $|0\rangle_{\text {in }}$ presentes na região $\mathrm{C}$ devemos determinar a relação entre esses modos. Para isso, precisamos evolui-los respectivamente para o futuro e para o passado de modo que o produto interno 
$\left(\nu_{\vec{k}^{\prime}}^{*}, u_{\vec{k}}\right)_{K G}$ possa ser calculado em alguma superfície de Cauchy. Faremos isso em etapas. Primeiramente, consideremos $u_{\vec{k}}$ e $\nu_{\vec{k}}$ respectivamente no início e no final da região $\mathrm{B}$, ou seja, $u_{\vec{k}}\left(t_{0}, \vec{x}\right)$ e $\nu_{\vec{k}}\left(t_{0}+T, \vec{x}\right)$, onde $t=t_{0}:=t_{S}-T / 2$ determina o início da fase instável B e $T$ sua duração na coordenada $t$. Da hipótese que o espaço-tempo é simétrico por reversão temporal em relação a $\Sigma_{t_{S}}$, segue (a menos de fases globais)

$$
u_{\vec{k}}\left(t_{S}-t, \vec{x}\right)=\nu_{-\vec{k}}^{*}\left(t_{S}+t, \vec{x}\right)
$$

Em particular, para $t=T / 2$ :

$$
\nu_{-\vec{k}}^{*}\left(t_{0}+T, \vec{x}\right)=u_{\vec{k}}\left(t_{0}, \vec{x}\right) .
$$

Decompondo $u_{\vec{k}}$ em termos da base $\left\{w_{\Omega}, v_{\alpha}, w_{\Omega}^{*}, v_{\alpha}^{*}\right\}$ [vide Eqs. 2.18), (3.5) e (3.8)] e substituindo na expressão acima, obtemos

$$
\begin{aligned}
\nu_{-\vec{k}}^{*}\left(t_{0}+T, \vec{x}\right)= & c_{\Omega \vec{k}}^{*} w_{\Omega}\left(t_{0}, \vec{x}\right)-d_{\Omega \vec{k}} w_{\Omega}^{*}\left(t_{0}, \vec{x}\right) \\
& +\int d \mu(\alpha)\left[c_{\alpha \vec{k}}^{*} v_{\alpha}\left(t_{0}, \vec{x}\right)-d_{\alpha \vec{k}} v_{\alpha}^{*}\left(t_{0}, \vec{x}\right)\right],
\end{aligned}
$$

onde, por simplicidade, assumimos a existência de um único auto-valor negativo $-\Omega^{2}$ na região B. Agora, usando a decomposição

$$
\begin{aligned}
u_{\vec{k}}\left(t_{0}+T, \vec{x}\right)= & c_{\Omega \vec{k}}^{*} w_{\Omega}\left(t_{0}+T, \vec{x}\right)-d_{\Omega \vec{k}} w_{\Omega}^{*}\left(t_{0}+T, \vec{x}\right) \\
& +\int d \mu(\alpha)\left[c_{\alpha \vec{k}}^{*} v_{\alpha}\left(t_{0}+T, \vec{x}\right)-d_{\alpha \vec{k}} v_{\alpha}^{*}\left(t_{0}+T, \vec{x}\right)\right]
\end{aligned}
$$

e as propriedades

$$
v_{\alpha}\left(t_{0}+T, \vec{x}\right)=\exp \left(-i \varpi_{\alpha} T\right) v_{\alpha}\left(t_{0}, \vec{x}\right)
$$

$\mathrm{e}$

$$
\begin{aligned}
w_{\Omega}\left(t_{0}+T, \vec{x}\right)= & 2 i \sinh (\Omega T-i \pi / 6) w_{\Omega}\left(t_{0}, \vec{x}\right) \\
& -2 i \sinh (\Omega T) w_{\Omega}^{*}\left(t_{0}, \vec{x}\right),
\end{aligned}
$$

podemos projetar $u_{\vec{k}}\left(t_{0}+T, \vec{x}\right)$ em $\nu_{\vec{k}}^{*}\left(t_{0}+T, \vec{x}\right)$, obtendo para valores de $\Omega T$ grandes 
o suficiente,

$$
\left(\nu_{\vec{k}^{\prime}}^{*}, u_{\vec{k}}\right)_{\mathrm{KG}} \sim e^{\Omega T} \zeta_{\vec{k} \vec{k}^{\prime}}
$$

onde

$$
\zeta_{\vec{k} \vec{k}^{\prime}}=i\left[\left(c_{\Omega \vec{k}}^{*} e^{-i \pi / 6}-d_{\Omega \vec{k}}\right) c_{\Omega-\vec{k}^{\prime}}-\left(c_{\Omega \vec{k}}^{*}-d_{\Omega \vec{k}} e^{i \pi / 6}\right) d_{\Omega-\vec{k}^{\prime}}^{*}\right]
$$

Com isso, o valor esperado da densidade de número de partículas (por elemento do espaço de fase) com números quânticos $\vec{k}^{\prime}$ presentes na região C é dado por

$$
\frac{d\left\langle N_{\vec{k}^{\prime}}\right\rangle}{d^{3} k^{\prime}} \sim e^{2 \Omega T} \int d^{3} k\left|\zeta_{\vec{k} \vec{k}^{\prime}}\right|^{2}
$$

que cresce exponencialmente com a duração da fase B. Note que essa produção de partículas ocorre em profusão (para $e^{2 \Omega T} \gg 1$ ) mesmo que as transições entre as fases A e B e entre as fases B e C sejam suficientemente lentas de modo a minimizar a produção de partículas devida à mudança na geometria de fundo $\left(d_{\Omega \vec{k}} \approx 0\right)$.

O quadro geral do efeito do despertar do vácuo vai se completando. Uma vez o efeito disparado, as flutuações de vácuo são exponencialmente amplificadas, juntamente com sua densidade de energia, até que a retro-ação desta última sobre o espaço-tempo de fundo (ou outra contribuição independente) guie a evolução subsequente do sistema a uma nova configuração de equilíbrio. Como consequência, as flutuações quânticas amplificadas são "liberadas" em forma de partículas do campo. Mas essa produção de partículas tem um custo energético, que só pode ser bancado pela energia gravitacional do sistema (lembrando que a energia total de vácuo se mantém constante enquanto as flutuações são exponencialmente amplificadas). Mas uma questão continua pendente: de que maneira as flutuações de vácuo agem sobre o espaço-tempo de fundo? 


\subsection{De flutuações quânticas a perturbações clás- sicas}

Pouco tempo depois de publicarmos os resultados do despertar do vácuo no contexto de objetos compactos, P. Pani et al. publicaram uma análise [17] mostrando uma interessante relação entre esse efeito quântico e um fenômeno clássico chamado escalarização espontânea 18,19]. Este último decorre do fato que, classicamente, a solução $\phi \equiv 0$ da equação de Klein-Gordon (2.2), na geometria de fundo de um objeto compacto, é instável para alguns valores de acoplamento $\xi$ e parâmetros do objeto compacto (massa e raio). Na verdade, a região do espaço de parâmetros para a qual tal instabilidade ocorre é a mesma para a qual o despertar do vácuo também é disparado. No caso do efeito clássico, pode-se mostrar que a região de instabilidade $\operatorname{com} \xi<0$ permite uma solução de campo $\phi$ estático e não-nulo de modo que a energia do sistema total (objeto compacto e campo $\phi \neq 0$ ) é menor do que a com configuração de campo $\phi$ nulo. Ou seja, na mesma região com $\xi<0$ em que o despertar do vácuo é disparado, é energeticamente favorável que a configuração clássica de campo assuma um valor diferente de zero. Daí o nome do efeito, em analogia com o fenômeno de magnetização espontânea.

Pani e seus colaboradores conjecturaram, então, que o despertar do vácuo funcionaria semeando as perturbações necessárias para que o campo nulo clássico evoluisse para o perfil não nulo mais estável. E, de fato, num trabalho publicado quase simultaneamente à nossa análise da criação de partículas que acompanha o processo de restabilização, M. Ruiz et al. mostraram que o processo de escalarização espontânea é acompanhado de intensa emissão de radiação escalar [20], que é a exata faceta clássica da nossa criação de partículas. No entanto, devemos ter em mente que a validade dessa análise energética é restrita à região $\operatorname{com} \xi<0$. Na região $\operatorname{com} \xi>0$, embora haja, no espaço de parâmetros, regiões de instabilidade da solução clássica $\phi \equiv 0$ (as mesmas nas quais o despertar do vácuo é disparado), as configurações 
"escalarizadas" possuem energia maior do que a de campo nulo. O destino final da instabilidade nesse caso ainda está sob investigação.

Por mais que pareça razoável o cenário em que o despertar do vácuo semeie perturbações clássicas que, então, evoluiriam para um perfil de campo clássico não nulo, há sutilezas que devem ser tratadas apropriadamente. Estamos, aqui, na interface entre os mundos quântico e clássico. Sob a ótica quântica, a instabilidade é responsável pelo crescimento das flutuações $\left\langle\Phi^{2}\right\rangle$ mas mantendo nulo o valor de $\langle\Phi\rangle$. Sob a ótica clássica, em algum momento as flutuações $\left\langle\Phi^{2}\right\rangle$ dão origem a uma perturbação clássica $\delta \phi \sim \sqrt{\left\langle\Phi^{2}\right\rangle}$. Mas para que essa "classicalização" ocorra é necessário que haja perda de coerência do estado do campo. Além disso, seria desejável que correlações clássicas surgissem entre $\Phi$ e $\Pi$, de modo que, de fato, pudéssemos interpretar que, em algum momento $t_{c}$ (com a perda da coerência quântica e o aparecimento de correlações clássicas), uma perturbação clássica $\left(\delta \phi\left(t_{c}, \vec{x}\right), \delta \pi\left(t_{c}, \vec{x}\right)\right)$ surgisse e funcionasse como condição inicial para a evolução subsequente do sistema. Na Ref. 21 mostramos que essas duas coisas de fato acontecem. O aparecimento de correlações clássicas se deve à própria evolução livre do modo instável $w_{\Omega}$, a qual age como uma "compressão" (squeezing) do estado de vácuo, fazendo com que o setor instável do campo $\Phi$ adquira uma correlação do tipo $\delta(\pi-\Omega \phi)$. Já a perda de coerência quântica se dá pela interação do modo instável com flutuações do campo gravitacional e, via estes, com os outros modos estáveis do campo $\Phi$. Esse "ambiente" faz com que o estado coerente do modo instável se torne uma mistura estatística, distribuída de acordo com as correlações clássicas mencionadas acima. O tempo característico dessa "classicalização" é da mesma ordem de grandeza do tempo necessário para que a densidade de energia de vácuo rivalizasse com as componentes clássicas de fundo. Sendo assim, quando a retro-ação do campo $\Phi$ se tornar inevitável, as flutuações de vácuo já terão dado lugar a perturbações clássicas. Isso é bastante conveniente, pois a retro-ação de flutuações quânticas sobre o espaço-tempo clássico de fundo é uma questão em aberto dentro do formalismo canônico de TQCEC. 


\section{Capítulo 4}

\section{Comentários finais}

Nesta tese procurei apresentar o efeito do despertar do vácuo da maneira mais clara possível, deixando de lado detalhes que julgo menos centrais ao entendimento do efeito (como o papel desempenhado por algumas idealizações, como esfericidade [22] e estaticidade [23]). A escolha por abordar exclusivamente esse tema ao invés de discorrer também sobre meus trabalhos em outras linhas de pesquisa se deve ao fato de que foi a descoberta e análise desse efeito que eu considero como atestado de minha independência científica, mesmo que logo tenhamos incluído colaboradores nessas análises (o que, no meu entender, foi e é bastante positivo).

Para finalizar esta tese, gostaria de mencionar algumas questões que ainda se colocam para serem exploradas no contexto do efeito do despertar do vácuo. De particular interesse, para mim, são (i) descobrir o destino final dos objetos compactos que despertam o vácuo de um campo $\operatorname{com} \xi>0$ (caso em que a escalarização espontânea não parece ser um mecanismo capaz de restabilizar o sistema), (ii) a identificação de outros possíveis cenários onde a densidade de energia de vácuo pudesse ser amplificada pelo campo gravitacional (como, talvez, cenários de interesse cosmológico) e (iii) verificar a possibilidade de o efeito do despertar do vácuo ocorrer para campos que sabemos existir, como o campo eletromagnético. Uma investigação na linha (i) vem sendo desenvolvida em colaboração com Raphael Santarelli (que 
acabou de assumir uma posição na UERJ). Já as frentes (ii) e (iii) constituem temas de pesquisa pelos quais eu tenho bastante apreço, embora os persiga de maneira mais solitária e, por isso mesmo, num horizonte de médio a longo prazo. 


\section{REFERÊNCIAS}

[1] R. Eisberg e R. Resnick, Física Quântica (Campus, Rio de Janeiro, 1988).

[2] O. Bratteli e D. W. Robinson, Operator Algebras and Quantum Statistical Mechanics, vols. 1 e 2 (Springer-Verlag, New York, 1981).

[3] H. B. G. Casimir, Proc. Kon. Ned. Akad. Wetensch. B51, 793 (1948).

[4] S. Lamoreaux, Phys. Rev. Lett. 78, 5 (1996).

[5] R. M. Wald, General Relativity (The University of Chicago Press, Chicago, 1984).

[6] K. W. Ford e J. A. Wheeler, Geons, Black Holes, and Quantum Foam: A Life in Physics (W. W. Norton \& Company, New York, 1998).

[7] N. D. Birrell e P. C. W. Davies, Quantum Field Theory in Curved Space (Cambridge University Press, Cambridge, 1982).

[8] S. A. Fulling, Aspects of Quantum Field Theory in Curved Space-Time (Cambridge University Press, Cambridge, 1989).

[9] R. M. Wald, Quantum Field Theory in Curved Spacetime and Black Hole Thermodynamics (The University of Chicago Press, Chicago, 1994).

[10] S. W. Hawking e G. F. R. Ellis, The Large Scale Structure of Spacetime (Cambridge University Press, Cambridge, 1973). 
[11] S. W. Hawking, Nature 248, 30 (1974); S. W. Hawking, Commun. Math. Phys. 43, 199 (1975).

[12] M. J. Radzikowski, The Hadamard Condition and Kay's Conjecture in (Axiomatic) Quantum Field Theory in Curved Spacetimes, tese de doutorado (Princeton University).

[13] W. C. C. Lima e D. A. T. Vanzella, Phys. Rev. Lett. 104, 161102 (2010).

[14] W. C. C. Lima , G. E. A. Matsas e D. A. T. Vanzella, Phys. Rev. Lett. 105, $151102(2010)$.

[15] J. M. Lattimer e M. Prakash, Astrophys. J. 550, 426 (2001).

[16] A. G. S. Landulfo, W. C. C. Lima, G. E. A. Matsas e D. A. T. Vanzella, Phys. Rev. D 86, 104025 (2012).

[17] P. Pani, V. Cardoso, E. Berti, J. Read e M. Salgado, Phys. Rev. D 83, 081501(R) (2011).

[18] T. Damour, G. Esposito-Farese, Phys. Rev. Lett. 70, 2220 (1993); Phys. Rev. D 54, 1474 (1996).

[19] T. Harada, Prog. Theor. Phys. 98, 359 (1997).

[20] M. Ruiz, J. C. Degollado, M. Alcubierre, D. Núñes e M. Salgado, Phys. Rev. D 86, 104044 (2012).

[21] A. G. S. Landulfo, W. C. C. Lima, G. E. A. Matsas e D. A. T. Vanzella, Phys. Rev. D 91, 024011 (2015).

[22] W. C. C. Lima, R. F. P. Mendes, G. E. A. Matsas e D. A. T. Vanzella, Phys. Rev. D 87, 104039 (2013).

[23] R. F. P. Mendes, G. E. A. Matsas e D. A. T. Vanzella, Phys. Rev. D 90, 044053 (2014). 